\title{
GLOBAL SOLVABILITY ON COMPACT NILMANIFOLDS OF THREE OR MORE STEPS
}

\author{
JACEK M. CYGAN AND LEONARD F. RICHARDSON
}

\begin{abstract}
We apply the methods of representation theory of nilpotent Lie groups to study the convergence of Fourier series of smooth global solutions to first order invariant partial differential equations $D f=g$ in $C^{\infty}$ of a compact nilmanifold of three or more steps. We investigate which algebraically welldefined conditions on $D$ in the complexified Lie algebra imply that smooth infinite-dimensional irreducible solutions, when they exist, satisfy estimates strong enough to guarantee uniform convergence of the irreducible (or primary) Fourier series to a smooth global solution. This extends and improves the results of an earlier two step paper.
\end{abstract}

0. Introduction and preliminaries. Let $N$ be a nilpotent Lie group, $\Gamma \backslash N$ a compact nilmanifold, and $D$ a differential operator, left-invariant on $N$, and viewed on $\Gamma \backslash N$. If $g \in C^{\infty}(\Gamma \backslash N)$ and if $g_{\pi}$ is the orthogonal component of $g$ in some irreducible subspace corresponding to the irreducible unitary representation $\pi$, then $g_{\pi} \in C^{\infty}(\Gamma \backslash N)$ too [2]. Modulo unitary equivalence, we may think of $g_{\pi}$ as being a $C^{\infty}$-vector in any concrete realization, or model, of $\pi$. We will determine algebraically well-defined conditions on first order and suitable higher order $D$ under which the global solvability of $D f=g$ in $C^{\infty}(\Gamma \backslash N)$ is equivalent to the solvability of $\pi(D) f_{\pi}=g_{\pi}$ in the $C^{\infty}$-vectors for each $\pi$ in the spectrum of $\Gamma \backslash N$. In one sense, we will be presenting algebraic conditions on $D$ for the reduction of a global (geometrical) problem on $\Gamma \backslash N$ to a collection of purely group (representation) theoretic problems, none of which needs to be regarded as living on the manifold $\Gamma \backslash N$. Operators $D$ admitting such a reduction are called globally regular (Definition (0.1)). In effect, we will prove global regularity for suitable operators by showing that if the smooth solutions $f_{\pi}$ of $\pi(D) f_{\pi}=g_{\pi}$ exist for each $\pi$, then they can be summed uniformly to a smooth global solution of $D f=g$. (Globally regular operators are usually neither locally solvable nor onto $C^{\infty}(\Gamma \backslash N)$ ! [12].) In order to make the necessary estimates on $f_{\pi}$, we construct a suitable Schrödinger model of $\pi$, which, for convenience, is not in the Hilbert space $L^{2}(\Gamma \backslash N)$.

In this paper, we extend to nilmanifolds of three or more steps (and correct) some of the results for two step nilmanifolds presented in [7].

In order to describe the main results, it will be helpful to review the classical situation on a torus $T^{2}$ of two dimensions (the situation being similar for $T^{n}$ with $n>2$ ). Let $D=\alpha \partial / \partial x+\beta \partial / \partial y$ and suppose, for simplicity, that $\alpha$ and $\beta$ are real.

Received by the editors July 24, 1984 and, in revised form, April 10, 1986.

1980 Mathematics Subject Classification (1985 Revision). Primary 22E27, 22E30; Secondary $22 \mathrm{E} 25$.

This research was supported in part by the National Science Foundation. 
Then $D$ is globally regular if and only if $\beta / \alpha$ is not a (transcendental) Liouville number. The problem with Liouville numbers is that, in solving for the Fourier transform of the solution function, small divisors occur. Now, every nilmanifold $\Gamma \backslash N$ contains the structure of a torus, $\Gamma[N, N] \backslash N$, although this torus does not reflect any of the nonabelian structure of $N$. The only representations in $(\Gamma \backslash N)^{-}$ which are not infinite dimensional are the one-dimensional characters of $\Gamma[N, N] \backslash N$. Since the presence of this torus is inescapable, we denote, for each $g \in C^{\infty}(\Gamma \backslash N)$, the sum of the one-dimensional components of $g$ by $g_{0}$. Then global regularity is informally taken to mean that the solvability of $D f=g$ is equivalent to the solvability of $\pi(D) f_{\pi}=g_{\pi}$ in $C^{\infty}$-vectors for each infinite-dimensional $\pi \in(\Gamma \backslash N)$, modulo the solvability of $D f_{0}=g_{0}$ in $C^{\infty}$ of the torus $\Gamma[N, N] \backslash N$. In order to state the formal definitions and summarize the new results, we need a few preliminaries.

Let $\mathfrak{N}$ denote a finite-dimensional real nilpotent Lie algebra, and $N=\exp \mathfrak{N}$ the corresponding Lie group. Then Malcev proved there exists a discrete subgroup $\Gamma \subset N$ which is cocompact, meaning that the nilmanifold $\Gamma \backslash N$ is a compact homogeneous space, of cosets of the form $\Gamma n$, if and only if $\mathfrak{N}$ has rational structure constants with respect to some suitable basis. Such a $\Gamma$ is never normal when $N$ is nonabelian. If $\Gamma$ exists, the rational basis of $\mathfrak{N}$ can be selected from $\log \Gamma$. If $\Gamma \backslash N$ is a compact nilmanifold, a subspace $V \subset \mathfrak{N}$, is called rational if and only if it is spanned by vectors which are finite linear combinations with rational coefficients of elements of a rational basis of $\mathfrak{N}$. A subgroup $M \subset N$ is called rational if and only if $\mathfrak{M}=\log M$ is a rational subspace of $\mathfrak{N}$, and this is equivalent to $\Gamma \cap M \backslash M$ being compact $[\mathbf{1 3}]$.

If $\Gamma \backslash N$ is a compact nilmanifold and $M$ is a normal rational subgroup of $N$, then Malcev proved the existence of one-parameter coordinate subgroups $d_{1}(t), \ldots, d_{k}(t)$, where $k=\operatorname{dim}(M \backslash N)$, with the following properties. If $N_{k}=M \rtimes d_{k}(\mathbf{R})$, a semidirect product with $M$ normal, and if $N_{i}=N_{i+1} \rtimes d_{i}(\mathbf{R})$, then $N_{1}=N$. Also, $d_{i}(n) \in \Gamma$ for each $n \in Z$, the integers $[\mathbf{1 3}]$.

Let $\hat{N}$ denote the space of equivalence classes of all irreducible unitary representations on $N$. Then Kirillov proved that the elements of $\hat{N}$ are in one-to-one correspondence with the so-called Kirillov orbits of $\operatorname{Ad}^{*} N$ acting in $\mathfrak{N}^{*}$, the linear dual of $\mathfrak{N}$. If $\pi \in \hat{N}$ corresponds to an orbit $O_{N}(\pi)=\left(\operatorname{Ad}^{*} N\right) \Lambda$, we may write $\pi=\pi_{\Lambda}$, and we may speak of $\Lambda \in \hat{N}$, for convenience. If $\Lambda \in \mathcal{O}_{N}(\pi)$, there is a subalgebra $\mathfrak{M} \subset \mathfrak{N}$, of maximal dimension so as to be subordinate to $\Lambda$, in the sense that $\Lambda([\mathfrak{M}, \mathfrak{M}])=0$. Then $\Lambda$ determines a character of $M=\exp \mathfrak{M}$ by $\chi_{\Lambda}(m)=\exp [i \Lambda(\log m)]$, and $\chi_{\Lambda}$ induces $\pi$, in the sense of Mackey. The Mackey induced representation space is

$$
\begin{aligned}
& \left\{f: N \rightarrow C \mid f(m n)=\chi_{\Lambda}(m) f(n),\right. \\
& \left.\quad \text { for all } m \in M, n \in N, \text { and }|f| \in L^{2}(M \backslash N)\right\},
\end{aligned}
$$

and $\pi_{\Lambda}(n)$ acts by right translation on this space.

The following definition, theorem, and proof are contained in [18, §3]. They are included here since we will refer later to certain details of the proof.

DEFINITION 0. Let $\mathfrak{N}$ be any nonabelian nilpotent Lie algebra and $\Lambda \in \mathfrak{N}^{*} \sim$ $\{0\}$. A maximal subordinate subalgebra $\mathfrak{M}_{\Lambda}$ for $\Lambda$ is called special if there is a decomposition $\mathfrak{N}=\mathbf{R} X_{1} \oplus \cdots \oplus \mathbf{R} X_{k} \oplus \mathfrak{M}_{\Lambda}$, where the vectors $X_{1}, \ldots, X_{k}$, called the external vectors, have the following properties. 
(i) $\mathfrak{N}_{j}=\mathbf{R} X_{j} \oplus \cdots \oplus \mathbf{R} X_{k} \oplus \mathfrak{M}_{\Lambda}$ is a subalgebra of $\mathfrak{N}$ for each $j=1, \ldots, k+1$, where by $\mathfrak{N}_{k+1}$ we mean $\mathfrak{M}_{\Lambda}$, and $\mathfrak{N}_{1}=\mathfrak{N}$.

(ii) For each $j=1, \ldots, k$, there is an ideal $\mathfrak{W}_{j}$ of $\mathfrak{\Upsilon}_{j}$ with $\Lambda\left(\mathfrak{W}_{j}\right)=\{0\}$, such that $\mathfrak{N}_{j} / \mathfrak{W}_{j}$ has 1-dimensional center, on which the image of $\Lambda$ is nontrivial.

(iii) For each $j=1, \ldots, k$ there is a vector $\bar{Y}_{j} \in \mathfrak{N}_{j} / \mathfrak{W}_{j}$ such that $\left[\bar{X}_{j}, \bar{Y}_{j}\right]$ spans the center of $\mathfrak{N}_{j} / \mathfrak{W}_{j}$, and the image $\overline{\mathfrak{M}}_{\Lambda}$ of $\mathfrak{M}_{\Lambda}$ lies in the centralize: $\mathfrak{Z}\left(\bar{Y}_{j}\right)$, which equals $\mathfrak{N}_{j+1}$. If $j=k$, we have $\overline{\mathfrak{M}}_{\Lambda}=\mathfrak{Z}\left(\bar{Y}_{k}\right)$.

THEOREM 0 . Let $\mathfrak{N}$ be any nilpotent Lie algebra and $\Lambda \in \mathfrak{N}^{*} \sim\{0\}$. Then there exists a special maximal subordinate subalgebra $\mathfrak{M}_{\Lambda}$ for $\Lambda$. If there exists a cocompact discrete subgroup $\Gamma \subset N=\exp \mathfrak{N}$, and if $\Lambda(\log \Gamma) \subset \mathbf{Q}$, the rational numbers, then $\mathfrak{M}_{\Lambda}$, the external vectors, and $Y_{1}, \ldots, Y_{k}$ (preimages of $\bar{Y}_{1}, \ldots, \bar{Y}_{k}$ ) can all be taken to be rational.

Proof. Let $\mathfrak{Z}(\mathfrak{A})$, where $\mathfrak{A}$ is an algebra, denote the center of $\mathfrak{A}$, whereas $\mathfrak{Z}(Y)$, where $Y$ is a vector, denotes the centralizer of $Y$. If $\mathfrak{N} / \operatorname{ker}(\Lambda \mid \mathfrak{Z}(\mathfrak{N}))$ has 1-dimensional center, then we let $\mathfrak{W}_{1}=\operatorname{ker}(\Lambda \mid \mathfrak{Z}(\mathfrak{N}))$. Otherwise, we factor out $\operatorname{ker}(\Lambda \mid \mathfrak{Z}(\mathfrak{N} / \operatorname{ker}(\Lambda \mid \mathfrak{Z}(\mathfrak{N}))))$, etc., until we obtain an ideal $\mathfrak{W}_{1} \subset \mathfrak{N}_{1}=\mathfrak{N}$ such that $\mathfrak{N}_{1} / \mathfrak{W}_{1}$ has 1-dimensional center, on which $\Lambda$ is nontrivial. The existence of $\mathfrak{W}_{1}$ is guaranteed by the fact that $\Lambda \neq 0$, and because $\operatorname{dim}(\mathfrak{N})<\infty$. If $\Lambda$ is rational, so is $\mathfrak{W}_{1}$.

By Kirillov's structure theorem $[\mathbf{1 2}]$, for nilpotent Lie algebras with 1-dimensional center, there are vectors $\bar{X}_{1}$ and $\bar{Y}_{1}$ in $\mathfrak{N}_{1} / \mathfrak{W}_{1}$ with $\bar{Y}_{1}$ in the next to the last stage of the lower central series of $\mathfrak{N}_{1} / \mathfrak{W}_{1}$, for which $\Lambda\left(\left[\bar{X}_{1}, \bar{Y}_{1}\right]\right) \neq 0$ (by abuse of notation, we regard $\Lambda$ as acting on $\left.\mathfrak{N}_{1} / \mathfrak{W}_{1}\right)$. By the Malcev structure theory [13], in the case of $\Lambda$ rational, we can pick the preimages $X_{1}$ and $Y_{1}$ rational as well. In either case, we can decompose $\overline{\mathfrak{N}}_{1}=\mathbf{R} \bar{X}_{1} \oplus \mathfrak{Z}\left(\bar{Y}_{1}\right)$, as in Kirillov [12]. We denote $\overline{\mathfrak{N}}_{2}=\mathfrak{Z}\left(\bar{Y}_{1}\right)$. If $\overline{\mathfrak{N}}_{2}$ is abelian, then $\mathfrak{N}_{2}=\mathfrak{M}_{\Lambda}$ the special maximal subordinate subalgebra. If not, we proceed as follows, noting that $\mathfrak{N}_{2}$ is automatically rational if $\Lambda$ and $Y_{1}$ are rational.

Next we find an ideal $\mathfrak{W}_{2}$ in $\mathfrak{N}_{2}, \mathfrak{W}_{2} \supset \mathfrak{W}_{1}$, just as we found $\mathfrak{W}_{1} \subset \mathfrak{N}_{1}$ above. In $\mathfrak{N}_{2} / \mathfrak{W}_{2}$, we find $\bar{X}_{2}$ and $\bar{Y}_{2}$ just as we found $\bar{X}_{1}$ and $\bar{Y}_{1}$ above. Thus we have $\mathfrak{N}_{2} / \mathfrak{W}_{2}=\mathbf{R} \bar{X}_{2} \oplus \mathfrak{Z}\left(\bar{Y}_{2}\right)$ and $\mathfrak{N}_{2}=\mathbf{R} X_{2} \oplus \mathfrak{N}_{3}$, where the preimages $X_{2}$ and $Y_{2}$ can again be taken to be rational in the case of rational $\Lambda$. If $\overline{\mathfrak{N}}_{3}$ is abelian, then $\mathfrak{N}_{3}=\mathfrak{M}_{\Lambda}$. If not, we continue this process, which must terminate in finitely many steps, since $\operatorname{dim}(\mathfrak{N})<\infty$. Now $\mathfrak{N}=\mathbf{R} X_{1} \oplus \cdots \oplus \mathbf{R} X_{k} \oplus \mathfrak{M}_{\Lambda}$, where $\mathfrak{M}_{\Lambda}$ is clearly subordinate to $\Lambda$. It remains only to show that $\mathfrak{M}_{\Lambda}$ is of maximal dimension so as to be subordinate to $\Lambda$.

If we denote $B_{\Lambda}(X, Y)=\Lambda([X, Y])$, then the dimension of a maximal subordinate subalgebra is always the dimension of the algebra minus $\frac{1}{2} \operatorname{rank}\left(B_{\Lambda}\right)$, and it is also always the dimension of the algebra minus $\frac{1}{2}$ the dimension of the coadjoint orbit. The maximality of $\overline{\mathfrak{M}}_{\Lambda}$ in $\overline{\mathfrak{N}}_{k}$ implies the maximality of $\mathfrak{M}_{\Lambda}$ in $\mathfrak{N}_{k}$, since ad $\mathfrak{W}_{k}$ does not enlarge the orbit. But now $\mathfrak{M}_{\Lambda}$ is maximal in $\mathfrak{N}_{k-1}$ also, since the dimension of the algebra is increased by 1 and the rank of $B_{\Lambda}$ is increased by 2. Hence, by induction, we conclude that $\mathfrak{M}_{\Lambda}$ is a special maximal subordinate subalgebra for $\Lambda$. This proves Theorem 0 .

We will denote by $(\Gamma \backslash N)$ the subspace of $\hat{N}$ occurring in the discrete direct sum decomposition of $L^{2}(\Gamma \backslash N)$. By the combined results of C. C. Moore [15] and the 
second author $[\mathbf{1 8}], \pi \in(\Gamma \backslash N)^{\wedge}$ if and only if if there is a rational $\Lambda \in \mathcal{O}_{N}(\pi)$, and a rational special maximal subordinate subalgebra $\mathfrak{M}_{\Lambda}$, such that $\Lambda: \log \left(\Gamma \cap M_{\Lambda}\right) \rightarrow$ Z. Henceforth, we consider only such rational $\Lambda$. In particular, $\Lambda: \log (\Gamma \cap \mathfrak{Z}(N)) \rightarrow$ Z. $(\mathfrak{Z}(N)$ is rational, by $[\mathbf{1 3}]$.) The multiplicity $m(\pi)$ with which $\pi$ occurs in the $\pi$-primary summand $\nVdash_{\pi} \subset L^{2}(\Gamma \backslash N)$ is known explicitly, but here we need only the fact that $m(\pi)<\infty[\mathbf{1 8}]$.

If $H_{\pi}=H_{\pi, 1} \oplus \cdots \oplus H_{\pi, m(\pi)}$ is an irreducible decomposition, the spaces $H_{\pi, j}$ are not canonical. Nevertheless, we will use irreducible decompositions because of the convenience of their Schrödinger models, which we will construct. If $g \in$ $L^{2}(\Gamma \backslash N)$, we will write $g=\sum_{\pi \in(\Gamma \backslash N)^{\wedge}} g_{\pi}$, the primary Fourier decomposition into components, and $g_{\pi}=\sum_{q=1}^{m(\pi)} g_{\pi, q}$ for some particular irreducible decomposition. Auslander and Brezin proved that, if $g \in C^{\infty}(\Gamma \backslash N)$, then each $g_{\pi, q} \in C^{\infty}(\Gamma \backslash N)$, which implies in turn that $g_{\pi} \in C^{\infty}(\Gamma \backslash N)$. Furthermore, the sums $g=\sum g_{\pi}=$ $\sum g_{\pi, q}$ both converge uniformly, if $g \in C^{\infty}(\Gamma \backslash N)$. Furthermore, if we begin with some functions $g_{\pi, q} \in H_{\pi, q}^{\infty}=H_{\pi, q} \cap C^{\infty}(\Gamma \backslash N)$, then $\sum_{\pi, q} g_{\pi, q}=g \in C^{\infty}(\Gamma \backslash N)$ if, and only if, $\sum_{\pi, q}\left\|U g_{\pi, q}\right\|_{2}^{2}<\infty$, for each fixed $U \in \mathcal{U}(\mathfrak{N})$, the universal enveloping algebra of $\mathfrak{N}[\mathbf{2}]$. If $D$ is a left-invariant differential operator on $N$, viewed on $\Gamma \backslash N$, it follows that $D f_{\pi}=g_{\pi}$ can be solved in $\nvdash_{\pi}^{\infty}$ if and only if $D f_{\pi, q}=g_{\pi, q}$ can be solved in $H_{\pi, q}^{\infty}$ for each irreducible component $g_{\pi, q}$ of the $\pi$-primary function $g_{\pi}$.

The following lemma is already known, but we include a proof for completeness.

LEMMA 1. Let $\mathfrak{N} \supset \mathfrak{N}_{1} \supset \ldots \supset \mathfrak{N}_{k} \supset\{0\}$ be the lower central series of a nilpotent Lie algebra $\mathfrak{N}$ of step 3 or more $(k \geq 2)$. Let $\Lambda \in \mathfrak{N}^{*}, \Lambda \mid \mathfrak{N}_{k} \neq 0$. Then there exists a maximal subordinate subalgebra $\mathfrak{M}$ for $\Lambda$ which contains $\mathfrak{N}_{k-1}$.

PROOF. Let $\mathfrak{M}$ be any maximal subordinate subalgebra for $\Lambda$. Let $Y \in \mathfrak{N}_{k-1} \sim$ $\mathfrak{M}$. There is $X \in \mathfrak{M}$ such that $[X, Y] \notin \operatorname{ker}(\Lambda)$, and by the Jacobi identity $X \notin$ $\mathfrak{N}_{1} \supseteq \mathfrak{N}_{k-1}$ (if $k \geq 2$ ). Since $\mathfrak{M}$ conains the center, the span $\langle\mathfrak{M}, Y\rangle$ is a subalgebra of $\mathfrak{N}$. Let $\mathfrak{M}^{\prime}=\mathfrak{Z}(Y)=$ centralizer of $Y$ in $\langle\mathfrak{M}, Y\rangle$ (modulo $\operatorname{ker}\left(\Lambda \mid \mathfrak{N}_{k}\right)$ ). Then $\mathfrak{M}^{\prime}$ has codim $=1$ in $\langle\mathfrak{M}, Y\rangle$, so $\operatorname{dim}\left(\mathfrak{M}^{\prime}\right)=\operatorname{dim}(\mathfrak{M})$, and $\mathfrak{M}^{\prime}$ is subordinate to $\Lambda$. If there is still a $Y^{\prime} \in \mathfrak{N}_{k-1} \sim \mathfrak{M}$, we note by Jacobi that $\left[\mathfrak{N}_{k-1}, Y^{\prime}\right]=0$, so $Y^{\prime}$ may also be added to $\mathfrak{M}^{\prime}$. Eventually, $\mathfrak{M}^{\prime} \supset \mathfrak{N}_{k-1}$.

LEMMA $1^{\prime}$. Under the hypothesis of Lemma 1 there exists a special maximal subordinate subalgebra $\mathfrak{M}$ for $\Lambda$ which contains $\mathfrak{N}_{k-1}$.

PROOF. We will retrace the proof of Theorem 0 , showing how to guarantee that $\mathfrak{M}_{\Lambda} \supset \mathfrak{N}_{k-1}$. Sincd $\Lambda \mid \mathfrak{N}_{k} \neq 0, \mathfrak{N} / \mathfrak{W}_{1}$ will still have a $k$-stage lower central series, where $\mathfrak{W}_{1}$ is as in the proof of Theorem 0 . (Caution: We are no longer using the symbol $\mathfrak{N}_{j}$ as in Theorem 0 , however.) Hence $Y_{1}$, chosen as in the proof of Theorem 0 , will also lie in $\mathfrak{N}_{k-1}$. Let $Z_{1}=\left[X_{1}, Y_{1}\right]$. By Jacobi's identity, $\mathfrak{Z}\left(\bar{Y}_{1}\right) \supset \overline{\mathfrak{N}}_{k-1}$. If $\overline{\mathfrak{N}}_{k-1} \neq \mathbf{R} \bar{Y}_{1}$, then there exists a $(k-1)$-fold bracket product $\bar{A} \in \mathfrak{Z}\left(\bar{Y}_{1}\right) \sim \mathbf{R} \bar{Y}_{\mathbf{1}}$. If $\bar{A}$ is in the center of $\mathfrak{Z}\left(\bar{Y}_{1}\right)$, then $A \in \mathbf{R} Z_{1} \oplus \mathfrak{W}_{2}$ constructed as in the proof of Theorem 0 . Otherwise, we simply choose $\bar{Y}_{2}=\bar{A}$, in the proof of Theorem 0 . Since $[\overline{\mathfrak{N}}, \bar{A}]$ is central in $\overline{\mathfrak{N}}$ and hence in $\mathfrak{Z}\left(\bar{Y}_{1}\right)$ too, Kirillov's Structure Theorem provides that $\mathfrak{Z}\left(\bar{Y}_{1}\right)=\mathbf{R} \bar{X}_{2}+\mathfrak{Z}(\bar{A})$, where $\mathfrak{Z}(\bar{A})$ is the centralizer in $\mathfrak{Z}\left(\bar{Y}_{1}\right)$. At the $j$ th stage of the construction, any remaining central elements of $\overline{\mathfrak{N}}_{k-1}$ will be in the corresponding $Z_{j}+\mathfrak{W}_{j}$, and any noncentral $\bar{A} \in \overline{\mathfrak{N}}_{k-1} \sim \mathbf{R} \bar{Y}_{1} \oplus \cdots \oplus \mathbf{R} \bar{Y}_{j-1}$ can be chosen to be $\bar{Y}_{j}$. This process cannot terminate until some lth stage at 
which $\overline{\mathfrak{N}}_{k-1} \subset \mathbf{R} \bar{Y}_{1} \oplus \cdots \oplus \mathbf{R} \bar{Y}_{l} \oplus \mathfrak{W}_{l} \oplus \mathbf{R} \bar{Z}_{1} \oplus \cdots \oplus \mathbf{R} \bar{Z}_{l}$. Since $\mathfrak{M}_{\Lambda}$ contains $Y_{1}, \ldots, Y_{k}, Z_{1}, \ldots, Z_{k}, \mathfrak{W}_{1}, \ldots, \mathfrak{W}_{k}$, as in the proof of Theorem 0 , we have $\mathfrak{M}_{\Lambda} \supset$ $\mathfrak{N}_{k-1}$. This proves Lemma $1^{\prime}$.

COROLlaRY. If $\mathfrak{N}$ is of step 3 , i.e. $\mathfrak{N} \supset \mathfrak{N}_{1} \supset \mathfrak{N}_{2} \supset\{0\}$, then there exists a (special) maximal subordinate $\mathfrak{M}$ for $\Lambda$ which contains $\mathfrak{N}_{1}=[\mathfrak{N}, \mathfrak{N}]$-the commutator of $\mathfrak{N}$-so that $\mathfrak{M}$ is an ideal in $\mathfrak{N}$.

Now let $\Gamma \backslash N$ be a compact nilmanifold and let $g \in C^{\infty}(\Gamma \backslash N)$. Let $g_{0}$ denote the sum of all the 1-dimensional primary components of $g$, so that $g_{0}$ really lives on the torus $\Gamma[N, N] \backslash N$. (On this torus, the phenomenon of small divisors is inescapable, but none of the nonabelian structure of $N$ is present here.) Let $\pi \in$ $(\Gamma \backslash N)^{\wedge}$ be infinite dimensional, and let $\varkappa_{\pi}=H_{\pi, 1} \oplus \cdots \oplus H_{\pi, m(\pi)}$ be an irreducible decomposition of the $\pi$-primary subspace of $L^{2}(\Gamma \backslash N)$. We write $g_{\pi}=g_{\pi, 1}+\cdots+$ $g_{\pi, m(\pi)}$ for the corresponding components of $g$, all of which are in

$$
C^{\infty}(\Gamma \backslash N) \cap \mathcal{H}_{\pi}=\mathcal{H}_{\pi}^{\infty}=H_{\pi, 1}^{\infty} \oplus \cdots \oplus H_{\pi, m(\pi)}^{\infty} .
$$

(0.1). DEFinition. A left-invariant differential operator $D$ is called globally regular on $\Gamma \backslash N$ if the following three conditions imply that the $f_{\pi, q}$ 's can be chosen, if necessary, in such a way that $\sum_{\pi \in(\Gamma \backslash N)^{\wedge}} \sum_{q=1}^{m(\pi)} f_{\pi, q}$ converges uniformly to a function $f \in C^{\infty}(\Gamma \backslash N)$ such that $D f=g$ :

(i) $g \in C^{\infty}(\Gamma \backslash N)$.

(ii) For each infinite-dimensional $\pi \in(\Gamma \backslash N)$ a and $j \in\{1, \ldots, m(\pi)\}$ there exists $f_{\pi, q} \in H_{\pi, q}^{\infty}$ such that $D f_{\pi, q}=g_{\pi, q}$.

(iii) There exists $f_{0} \in C^{\infty}(\Gamma[N, N] \backslash N)$ such that $D f_{0}=g_{0}$.

Note that the function $g_{\pi, q} \in H_{\pi, q}$ can just as well be regarded as a $C^{\infty}$-vector $\tilde{g}_{\pi, q}$ in any realization of the irreducible representation $\pi$. From this viewpoint, if $D$ is globally regular, then the solution in $C^{\infty}(\Gamma \backslash N)$ of $D f=g \in C^{\infty}(\Gamma \backslash N)$ is reduced (except for the problem on the torus $\Gamma[N, N] \backslash N$ ) to the solution of $\pi(D) \tilde{f}_{\pi, q}=\tilde{g}_{\pi, q}$ in the $C^{\infty}$-vectors of the chosen realization of $\pi$. Thus the global geometrical problem on $\Gamma \backslash N$ is replaced by a purely group (representation) theoretic problem (together with a well-understood classical problem on a torus). This viewpoint is also critical to the proofs we present here since we will obtain our estimates on the $f_{\pi, q}$ by solving $\pi(D) \tilde{f}_{\pi, q}=\tilde{g}_{\pi, q}$ in a suitable Schrödinger model of $\pi$. The freedom of choice of this model grants us the flexibility needed to obtain our estimates.

Note that global regularity carries no implication that $D$ is onto $C^{\infty}(\Gamma \backslash N)$. (See [19].) Global regularity means only that if $\pi(D) \tilde{f}_{\pi, q}=\tilde{g}_{\pi, q}$ can be solved in the $C^{\infty}$-vectors for $\pi$, for each component $g_{\pi, q}$ of $g \in C^{\infty}(\Gamma \backslash N)$, and if $D f_{0}=g_{0}$ can be solved in $C^{\infty}$ of the torus, then $D f=g$ can be solved in $C^{\infty}(\Gamma \backslash N)$.

It follows from the definition of global regularity that suitable compositions of finitely many globally regular operators are again globally regular.

Proposition 1. Let $D=D_{n} \circ D_{n-1} \circ \cdots \circ D_{1}$ be a composition of $n$ globally regular operators having the property that $\operatorname{ker}\left(D_{i+1}\right) \subseteq \operatorname{range}\left(D_{i} \circ D_{i-1} \circ \cdots \circ D_{1}\right)$, $i=1, \ldots, n-1$. Then $D$ is globally regular too.

ProOF. It will suffice to give the proof for $n=2$. Suppose, for each $\Lambda \in(\Gamma \backslash N)$, there is a smooth solution $f_{\Lambda}$ to $D f_{\Lambda}=g_{\Lambda}$, where $\sum_{\Lambda} g_{\Lambda}=g \in C^{\infty}(\Gamma \backslash N)$. 
Then, for each $\Lambda, D_{2}\left(D_{1} f_{\Lambda}\right)=g_{\Lambda}$ and, by hypothesis, there exists $h_{\Lambda} \in \operatorname{ker}\left(D_{2}\right)$ such that $D_{2}\left(h_{\Lambda}+D_{1} f_{\Lambda}\right)=g_{\Lambda}$ and $\sum_{\Lambda}\left(h_{\Lambda}+D_{1} f_{\Lambda}\right)=h \in C^{\infty}(\Gamma \backslash N)$. Then there exists $k_{\Lambda} \in C^{\infty}(\Gamma \backslash N)$ such that $D_{1} k_{\Lambda}=h_{\Lambda}+D_{1} f_{\Lambda}$, and, since $D_{1}$ is globally regular, there exists $k_{\Lambda}^{\prime} \in C^{\infty}$ such that $\sum_{\Lambda} k_{\Lambda}^{\prime}=k \in C^{\infty}(\Gamma \backslash N)$ and $D k_{\Lambda}=D_{2}\left(h_{\Lambda}+D_{1} f_{\Lambda}\right)=g_{\Lambda}$ for each $\Lambda$. This proves the proposition.

We remark that the range of each $D_{i}$ seems to be very large-nearly all of $C^{\infty}$, as indicated by the examples in $[\mathbf{1 9}]$.

Our theorems about global regularity will be stated for $D=X+i Y$ in the complexified Lie algebra $\mathfrak{N}_{\mathbf{C}}$. Then many compositions of globally regular first order operators will again be globally regular.

Let $V$ be a real (finite-dimensional) vector space and $\mathbf{E}=\left\{e_{1}, \ldots, e_{n}\right\}$ a basis of $V$. Then $\alpha=\alpha_{1} e_{1}+\cdots+\alpha_{n} e_{n}$ is called a non-Liouville vector in $V$ with respect to $\mathbf{E}$ if there exist positive constants $d$ and $C$ such that

$$
|k \cdot \alpha|^{-1}=\left|k_{1} \alpha_{1}+\cdots+k_{n} \alpha_{n}\right|^{-1} \leq C\left(k_{1}^{2}+\cdots+k_{n}^{2}\right)^{d}=C|k|^{d}
$$

for all integers $k_{1}, \ldots, k_{n}$ such that $k_{1} \alpha_{1}+\cdots+k_{n} \alpha_{n} \neq 0$.

REMARK 1. If $\alpha \in V$ is a non-Liouville vector with respect to a basis $\mathbf{E}$ of $V$, then $\alpha$ is also a non-Liouville vector with respect to any basis $\mathbf{E}^{\prime}$ of $V$ rationally equivalent to $\mathbf{E}$.

ProOF. Let $\mathbf{E}=\tilde{Q} \mathbf{E}^{\prime}=q^{-1} Q \mathbf{E}^{\prime}$, where $\tilde{Q}$ is a rational matrix and $Q$ is an integer matrix such that $q \tilde{Q}=Q$ for some integer $q$. Then $\left|Q^{T} \alpha \cdot k\right|=|\alpha \cdot Q k| \geq$ $C|Q k|^{-d} \geq C^{\prime}|k|^{-d}$.

If a compact nilmanifold $\Gamma \backslash N$ has been specified, we call a vector in $\mathfrak{N}$ nonLiouville if it is non-Liouville with respect to any (every) rational basis selected from $\log \Gamma$.

As in $[\mathbf{2 1}$, p. 18] one can show that the set of all Liouville vectors (= the complement of the set of all non-Liouville vectors) has Lebesgue measure zero in $\mathfrak{N} \simeq \mathbf{R}^{n}$.

We call a vector in $\mathfrak{N}$ algebraic if and only if it can be expressed as a linear combination of vectors in $\log \Gamma$ with coefficients which are algebraic numbers.

Of course, every algebraic vector is non-Liouville (see e.g. [3, p. 79]). (Note that rational vectors are non-Liouville under our definition above, since we require $|k \cdot \alpha|^{-1}$ to be polynomially bounded only when $k \cdot \alpha \neq 0$.)

(0.2). Definition. $X \in \mathfrak{N}$ is said to have the non-Liouville (resp. algebraic, rational) supplementation property (relative to $\Gamma$ ) if for each stage $\mathfrak{N}_{j}$ of the lower central series of $\mathfrak{N}, \operatorname{ad}_{X} \mathfrak{N}_{j}$ contains a finite set $Z_{1}^{j}, \ldots, Z_{m}^{j}$ of non-Liouville (resp. algebraic, rational) vectors relative to $\log \Gamma$ such that every rational subspace $V$ of $\mathfrak{N}_{j+1}$ of codimension 1 in $\mathfrak{N}_{j+1}$ can be complemented to $\mathfrak{N}_{j+1}$ by some $Z_{i}^{j}$, $1 \leq i \leq m$.

REMARK 2. The rational supplementation property simply means that $\operatorname{ad}_{X}$ maps each stage of the lower central series of $\mathfrak{N}$ onto the next.

Clearly, there are Liouville vectors that have the rational supplementation property. On the other hand, we have the following

PROPOSITION 2. Let $X_{1}, \ldots, X_{n}$ Lie-generate a rational nilpotent Lie algebra $\mathfrak{N}$. If $X=\alpha_{1} X_{1}+\cdots+\alpha_{n} X_{n}$ is a non-Liouville vector (relative to the basis $\mathbf{B}$ of $\mathfrak{N}$ consisting of $X_{1}, \ldots, X_{n}$, and a sufficient set of their commutators) such that $\left\{\alpha_{1}, \ldots, \alpha_{n}\right\}$ is linearly independent over $\mathbf{Q}$, then $X$ has the non-Liouville supplementation property (relative to the discrete subgroup generated by $\exp B$ ). 
ProOF. Fix $r \leq k=$ length of the lower central series. Let the $r$-fold bracket products, $\Xi_{l}=\left[X_{i_{l}}\left[X_{j_{l}}\left[X_{k_{l}} \cdots\right]\right]\right], l=1, \ldots, m$, be a basis of $\mathfrak{N}_{r}$. Let $V=$ (linear) span of $\left\{\sum_{l=1}^{m} \xi_{l s} \Xi_{l}, 1 \leq s \leq m-1\right.$, all $\left.\xi_{l s} \in \mathbf{Q}\right\}$ be a rational subspace of $\mathfrak{N}_{r}$ of codimension 1 . Then the $m-1$ by $m$ matrix $\left(\xi_{l s}\right)$ is of rank $m-1$. Let $s_{0}$ be the number of a column the algebraic complement of which in $\left(\xi_{l_{s}}\right)$ is of full rank. Let $Z_{s_{0}} \in \mathfrak{N}_{r}$ be defined by replacing $X_{i_{s_{0}}}$ in the definition of $\Xi_{s_{0}}$ by the vector $X$ :

$$
Z_{s_{0}}=\left[X\left[X_{j_{s_{0}}}\left[X_{k_{s_{0}}} \cdots\right]\right]\right]=\sum_{i=1}^{n} \alpha_{i}\left[X_{i}\left[X_{j_{s_{0}}}\left[X_{k_{s_{0}}} \cdots\right]\right]\right]=\sum_{l=1}^{m} \beta_{l} \Xi_{l} .
$$

We notice that $\beta_{s_{0}}=\alpha_{i_{s_{0}}}+$ a rational linear combination of the other $\alpha_{i}$ 's; hence $\beta_{s_{0}} \neq 0$. Also, none of the formulas for $\beta_{l}, l \neq s_{0}$, resulting from (0.3), contains any term with $\alpha_{i_{s_{0}}}$. In order to prove that $\mathbf{R} Z_{s_{0}} \oplus V=\mathfrak{N}_{r}$, we will show that the $m$ by $m$ matrix obtained by augmenting $\left(\xi_{l s}\right)$ by a row consisting of $\beta_{1}, \ldots, \beta_{m}$ is of rank $m$. Indeed, the determinant of this matrix, when expanded along the row of $\beta_{j}$ 's, is of the form

$$
\begin{aligned}
(-1)^{j_{0}+1} \operatorname{det}\left[\left(\xi_{l s}\right)_{s \neq s_{0}}\right] \beta_{s_{0}}+\sum_{p \neq s_{0}}(-1)^{p+1} \operatorname{det}\left[\left(\xi_{l s}\right)_{s \neq p}\right] \beta_{j} \\
=(-1)^{j_{0}+1} \operatorname{det}\left[\left(\xi_{l s}\right)_{s \neq s_{0}}\right] \alpha_{i_{s_{0}}} \\
\quad+\text { a rational linear combination of the } \alpha_{i} \text { 's with } i \neq i_{s_{0}} .
\end{aligned}
$$

This is nonzero, since the $\alpha_{j}$ 's are linearly independent over $\mathbf{Q}$, and the coefficient of $\alpha_{i_{s_{0}}}$ is $\neq 0$. As in the proof of Remark 1 we can show now that $Z_{s_{0}}$ is a nonLiouville vector. Thus $Z_{s_{0}}, s_{0}=1, \ldots, m$, as defined by $(0.3)$ is the finite set of non-Liouville vectors of Definition (0.2) complementing every rational subspace $V$ of codim 1 in $\mathfrak{N}_{r}$.

Now we are ready to summarize the new results in this paper. In Theorem $1(\S 1)$ we prove that every real field with the (non-Liouville or rational) supplementation property (Definition (0.2)) is globally regular on every compact nilmanifold. This confirms an informal conjecture made by Roger Howe. In Theorem $2(\S 1$ ) we show that if $X$ is a non-Liouville vector field in the next to the last step of the lower central series of $\mathfrak{N}$, then $D=X$ is globally regular. The hypothesis that $X$ be non-Liouville cannot be relaxed as Example 1 shows. Example 2 illustrates some of the obstructions that one encounters in dealing with an arbitrary non-Liouville vector field $X$. All the other theorems, in $\S \S 2-4$, deal with complex vector fields $D=X+i Y$ with $X, Y \in \mathfrak{N},[X, Y]$ central (or 0$)$. Thus in Theorem $\mathbf{1}_{\mathbf{C}}(\S 2)$ we prove that $D$ is globally regular if $X$ and $Y$ commute and have property $\left(P_{k}\right)$, $k=1, \ldots, n$ (see $(2.6)$ ), which is a stronger hypothesis than the requirement that both $X$ and $Y$ have (non-Liouville) supplementation. Example 4 of $\S 2$ explains that such a stronger requirement is in fact needed. Theorem $2_{\mathbf{C}}(\S 2)$ states that $D$ is globally regular if both $X$ and $Y$ are non-Liouville vectors and are in the next to the last step of the lower central series of a metabelian algebra $\mathfrak{N}$. For 3-step (and lower) compact nilmanifolds $\Gamma \backslash N$, we show in Theorems 3 and 4 ( $\S \S 3$ and 4) that $D$ is globally regular if $X$ has the (non-Liouville) supplementation property, $Y$ is a non-Liouville vector and is in the next to the last step of the lower central series of $\mathfrak{N}$ (which is the commutator $[\mathfrak{N}, \mathfrak{N}]$ if $\mathfrak{N}$ is of step 3 , and $\mathfrak{N}$ itself if $\mathfrak{N}$ is of step 2) provided $[X, Y]$ (which then has to be central) is a non-Liouville vector 
and some commutativity relations between $X$ and $\mathfrak{N}$ and $Y$ and $\mathfrak{N}$ hold. In the case of a step 3 algebra $\mathfrak{N}$ and a noncentral $[X, Y]$ there is an example, suggested to us by L. Corwin, in which $D$ is not globally regular, even though $X$ has the supplementation property and $Y$ is an algebraic vector $\notin[\mathfrak{N}, \mathfrak{N}]$. The key thing there is that $X$ and $Y$ generate a step 3 subalgebra of $\mathfrak{N}$.

We elaborate upon that example in $\S 5$. It would be interesting to determine, in that context, what happens if $X$ and $Y$ generate a step 2 subalgebra of $\mathfrak{N}$. We are unable to settle this question. Example 2(c) of $\S 5$ indicates that $\pi_{\Lambda}(D)$ then is closely related to the not everywhere locally solvable operator $\partial / \partial x+i x \partial / \partial y$.

If $\mathfrak{N}$ is of step 2 , the above mentioned theorems yield the following three conditions, each of which alone is sufficient to imply that $D=X+i Y$ is globally regular on every step 2 compact nilmanifold $\Gamma \backslash N$.

$1^{\circ} . X$ and $Y$ satisfy $\left(P_{1}\right)$ (Corollary 2 of $\left.\S 4\right)$.

$2^{\circ}$. $X$ has the non-Liouville supplementation property, and both $Y$ and $[X, Y]$ are non-Liouville vectors (Theorem $4, \S 4$ ).

$3^{\circ}$. All the 3 vectors $X, Y$ and $[X, Y]$ are non-Liouville or, in particular, both $X$ and $Y$ are algebraic vectors (Corollary 1 of $\S 4$ ).

The relevance of the above step 2 theorems to what has been stated in our step 2 paper [7] is as follows: Statements $1^{\circ}$ and $2^{\circ}$ correct Theorems (3.5) and (3.13); statement $3^{\circ}$ is new. To be more specific, statement $2^{\circ}$ is precisely what is called Case I of Theorem (3.5) there (p. 542) when $Y=0$. We obtain the same result here by different method. Case II of Theorem (3.5) when $Y$ is central is still statement $2^{\circ}$ and then the assumption, necessary as Example 1 of $\S 1$ here demonstrates, that " $Y$ is algebraic (or non-Liouville)" is missing there. In Case III, $X, Y \notin[\mathfrak{N}, \mathfrak{N}]$ the assumption of Theorems (3.5) and (3.13) there that " $D \in \mathfrak{N}_{\mathbf{C}}$ has the supplementation property" meaning $[X, \mathfrak{N}]+[Y, \mathfrak{N}]=[\mathfrak{N}, \mathfrak{N}]$ requires the additional hypothesis that $X$ be algebraic (or non-Liouville) in case $[X, \mathfrak{N}] \neq[\mathfrak{N}, \mathfrak{N}]$ and that $Y$ be algebraic (or non-Liouville) too in case $[Y, \mathfrak{N}] \neq[\mathfrak{N}, \mathfrak{N}]$, as can be seen from Example 1 again. Also our proofs here correct an error in Cases II and III of Theorem (3.5). There the exponential function in the integrand did not behave as claimed in all cases. Thus if $[X, \mathfrak{N}] \neq[\mathfrak{N}, \mathfrak{N}]$ and $[Y, \mathfrak{N}] \neq[\mathfrak{N}, \mathfrak{N}]$ we need the assumption that both $X$ and $Y$ are algebraic (or non-Liouville) and then we do not need any supplementation! However, we still need $[X, Y]$ to be algebraic or non-Liouville (statement $3^{\circ}$ ). If, on the other hand, $[X, \mathfrak{N}]=[\mathfrak{N}, \mathfrak{N}]$ and $[Y, \mathfrak{N}] \neq[\mathfrak{N}, \mathfrak{N}]$ (or resp. $[Y, \mathfrak{N}]=[\mathfrak{N}, \mathfrak{N}]$ and $[X, \mathfrak{N}] \neq[\mathfrak{N}, \mathfrak{N}]$ ) we need $Y$ (or resp. $X$ ) to be non-Liouville, statement $2^{\circ}$, and then again we need to assume that $[X, Y]$ is algebraic (or non-Liouville). Finally, if both $[X, \mathfrak{N}]=[\mathfrak{N}, \mathfrak{N}]$ and $[Y, \mathfrak{N}]=[\mathfrak{N}, \mathfrak{N}]$ we do not need either $X$ or $Y$ to be algebraic, but we still need a stronger hypothesis to prove that $D$ is globally regular (see Example 2 of $\S 2$ ). The stronger hypothesis we assume is that $X$ and $Y$ satisfy $\left(P_{1}\right)$, and of course that $[X, Y]$ is non-Liouville-statement $1^{\circ}$.

As for the methods of proofs we use throughout the paper, roughly speaking, the idea is this. " $X$ having the supplementation property" implies $\pi_{\Lambda}(X)=\partial / \partial x$ in a suitably chosen Schrödinger model for $\pi_{\Lambda} \in(\Gamma \backslash N)$. This differentiation can of course be converted into a multiplication by $i \xi$ by means of a partial Fourier transform. " $X$ being in the next to the last step of the lower central series of $\mathfrak{N}$ " 
implies $\pi_{\Lambda}(X)=i \lambda x$ (again in a suitable model for $\pi_{\Lambda}$ ), that is we embed in $\mathfrak{N}$ a 3 $\operatorname{dim}$ Heisenberg algebra spanned by $\left\{X_{1}, Y_{1}, Z_{1}\right\}$ with either $X$ identified with $X_{1}$ or with $Y$ identified with $Y_{1}$. If now $D=X+i Y$ is a complex vector field, $[X, Y]=0$, and some extra commutativity conditions on $\mathfrak{N}$ hold, the two variables $x$ of $\pi_{\Lambda}(X)$ and $y$ of $\pi_{\Lambda}(Y)$ are in fact separated, i.e. a 5-dim Heisenberg algebra spanned by $\left\{X_{1}, X_{2}, Y_{1}, Y_{2}, Z\right\}$ can be embedded in $\mathfrak{N}$ with either $X$ identified with $X_{1}$ and $Y$ identified with $Y_{2}$ (Theorem 3) or with $X$ identified with $X_{1}$ and $Y$ identified with $X_{2}$ (Theorem $1_{C}$ ) or with $X$ identified with $Y_{1}$ and $Y$ identified with $Y_{2}$ (Theorem $2_{\mathbf{C}}$ ), and $\pi_{\Lambda}(D)$, acting on $\mathbf{H}_{\Lambda} \simeq L^{2}\left(\mathbf{R}^{p}\right)$ can be viewed as a multiplication by a polynomial in two variables. In our problems of global regularity of $D$ we deal with the estimates in terms of the Sobolev norms of some Schwartz functions divided by polynomials. The way those estimates depend on $\Lambda \in(\Gamma \backslash N)^{\wedge}$ turns out to be essential for our problem. If on the other hand $[X, Y] \neq 0$ is central, $\pi_{\Lambda}(D)$ can be reduced to the form $\partial / \partial x-\lambda x$ rather than a multiplication by a polynomial, which again corresponds to embedding in $\mathfrak{N}$ a 3 -dim Heisenberg algebra spanned by $X, Y$ and $[X, Y]$. Using appropriate integral estimates adapted from [7] we can show global regularity of $D$.

We are happy to thank L. Corwin, F. Greenleaf, R. Fabec and L. Baggett for their helpful suggestions and comments on the subject of this paper. F. Greenleaf, in particular, found a substantial error in an earlier version.

\section{Real fields on $n$-step nilmanifolds.}

THEOREM 1. Let $N$ be any 1-connected nilpotent Lie group possessing a cocompact discrete subgroup $\Gamma[\mathbf{1 3}]$. If $X \in \mathfrak{N}$ has the non-Liouville (or rational) supplementation property (relative to $\Gamma$ ), then $D=X$ is globally regular on $\Gamma \backslash N$.

ProOF. Let $\pi \in(\Gamma \backslash N)$, the set of irreducible unitary representations of $N$ in the spectrum of $\Gamma \backslash N$. The proof is accomplished by establishing inductively that, independent of global solvability questions, $\pi(D)$ satisfies certain Sobolev-

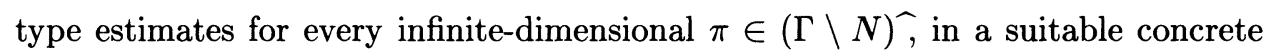
model of $\pi$. It is the formula for the estimates that is treated inductively, not the global regularity. Let $\Lambda$ be any member of the Kirillov orbit of $\pi$ which is rational on $\log \Gamma$ and integral on the center $[\mathbf{1 3}]$. (The theorem is already known for 2-step groups-Case I of Theorem (3.5) of [7].) If $\Lambda$ were trivial on the center $\mathfrak{Z}$, then we could factor $\mathfrak{Z}$ out - it is easy to see that $\pi(D)$ would not be affected by such a factoring process. So we can assume without loss of generality that $\Lambda$ is nontrivial on $\mathfrak{Z} \cap \mathfrak{N}_{k}$. Let $Z_{1}, \ldots, Z_{m}$ be the set of non-Liouville vectors in ad $\mathfrak{N}_{k-1}$ described in Definition (0.2). Then there is some $1 \leq j \leq m$ such that $\Lambda\left(Z_{j}\right)=\lambda_{j} \neq 0$. We remark that $Z_{j}=\alpha_{1} e_{1}+\cdots+\alpha_{n} e_{n}$ for a fixed rational basis $e_{1}, \ldots, e_{n}$ of $\mathfrak{N}_{k}$, and there are constants $C>0$ and (integer) $d>0$ (independent of $\Lambda$ ) such that

$$
\left|\lambda_{j}\right|^{-1}=\left|\Lambda\left(Z_{j}\right)\right|^{-1} \leq C\left(\Lambda\left(e_{1}\right)^{2}+\cdots+\Lambda\left(e_{n}\right)^{2}\right)^{d}
$$

for all (integral on $\left.\mathfrak{N}_{k}\right) \Lambda \in(\Gamma \backslash N) \hat{)} \Lambda\left(Z_{j}\right) \neq 0$. (If $D$ simply had rational supplementation, we would have picked a rational basis $Z_{1}, \ldots, Z_{r}$ of $\mathfrak{N}_{k}$ rather than the set $Z_{1}, \ldots, Z_{m}$ of non-Liouville vectors. Then we could replace (1.1) by

$$
\left|\lambda_{j}\right|^{-1} \leq M_{j},
$$


for some suitable constant $M_{j}$, using the fact that the set $\left\{\Lambda\left(Z_{j}\right) \mid \pi_{\Lambda} \in(\Gamma \backslash N) \widehat{\uparrow}, \Lambda\right.$ integral on $\mathfrak{Z}$, and $\left.\Lambda\left(Z_{j}\right) \neq 0\right\}$ is bounded away from 0 .)

By hypothesis, there is a vector $Y_{j}$ in the next to the last stage of the lower central series of $\mathfrak{N}$ such that $\left[X, Y_{j}\right]=Z_{j}$. Let $\overline{\mathfrak{N}}=\mathfrak{N} / \operatorname{ker}(\Lambda \mid \mathfrak{Z})$ if it has 1 -dim center, or $\mathfrak{N} / \mathfrak{W}_{1}$ as in the beginning of the proof of Theorem $0, \S 0$, otherwise; so $\left[\bar{X}, \bar{Y}_{j}\right]=\bar{Z}_{j}$, and $\overline{\mathfrak{N}}=\mathbf{R} \bar{X} \oplus \mathfrak{Z}\left(\bar{Y}_{j}\right)$, where $\mathfrak{Z}\left(\bar{Y}_{j}\right)$ is the centralizer of $\bar{Y}_{j}$. Without loss of generality, we can take a maximal subordinate subalgebra $\mathfrak{M}_{\Lambda}$ for $\Lambda$ such that $\overline{\mathfrak{M}}_{\Lambda}=\mathbf{R} \bar{Z}_{j} \oplus \mathbf{R} \bar{Y}_{j} \oplus \mathbf{R} \bar{X}_{1} \oplus \cdots \oplus \mathbf{R} \bar{X}_{i}$, and $\mathfrak{Z}\left(\bar{Y}_{j}\right)=\overline{\mathfrak{M}}_{\Lambda} \oplus \mathbf{R} \bar{X}_{i+\mathbf{1}} \oplus \cdots \oplus \mathbf{R} \bar{X}_{n}$, where the basis vectors $X_{1}, \ldots, X_{n}$ are chosen at our convenience and fixed. Pass one-parameter coordinate subgroups through $\exp \bar{Z}_{j}, \exp \bar{Y}_{j}, \exp \bar{X}$, and $\exp \bar{X}_{i}$, $i=1, \ldots, n$, and we may write

$$
\bar{N}=\bar{M}_{\Lambda} \rtimes \exp \left(\mathbf{R} \bar{X}_{i+1}\right) \rtimes \cdots \rtimes \exp \left(\mathbf{R} \bar{X}_{n}\right) \rtimes \exp (\mathbf{R} \bar{X}),
$$

a sequence of nested semidirect products [18]. In the corresponding Schrödinger model of $\pi$ in $L^{2}\left(\mathbf{R}^{n+1-i}\right)$, we have $\pi(\bar{D})=\pi(\bar{X})=\partial / \partial t, \pi\left(\bar{Y}_{j}\right)=i \lambda_{j} t$, and $\pi\left(\bar{Z}_{j}\right)=i \lambda_{j}$, where $t$ denotes the coordinate in $\exp (\mathbf{R} \bar{X})$. Taking the Euclidean partial Fourier transform ${ }^{\wedge}$ with respect to the variable $t$ we have

$$
\hat{X}=\hat{\pi}(\bar{X})=i \xi \quad \text { and } \quad \hat{Y}_{j}=\hat{\pi}\left(\bar{Y}_{j}\right)=-\lambda_{j} \frac{\partial}{\partial \xi}
$$

where $\xi$ denotes the dual variable of $t$.

Let the component of $g \in C^{\infty}(\bar{\Gamma} \backslash \bar{N})$ in the $q$ th irreducible $\pi$-subspace (noncanonical) be denoted $g_{\Lambda, q} \in C^{\infty}(\bar{\Gamma} \backslash \bar{N})$. Let $\tilde{g}_{\Lambda, q}$ denote the corresponding Schwartz function in $L^{2}\left(\mathbf{R}^{n+1-i}\right)$, and $\hat{g}_{\Lambda, q}$ the (Euclidean) partial Fourier transform of the Schwartz function $\tilde{g}_{\Lambda, q}[\mathbf{2 1}]$. If there is an $f_{\Lambda, q} \in C^{\infty}(\Gamma \backslash N)$ such that $D f_{\Lambda q}=g_{\Lambda, q}$, or $\tilde{D} \tilde{f}_{\Lambda, q}=\tilde{g}_{\Lambda, q}$, then

$$
\hat{D} \hat{f}_{\Lambda, q}=\hat{g}_{\Lambda, q}
$$

and

$$
\hat{f}_{\Lambda, q}\left(s_{\Lambda}, \xi\right)=\hat{g}_{\Lambda, q}\left(s_{\Lambda}, \xi\right)(i \xi)^{-1}
$$

is the unique Schwartz solution of $(1.2)$, where the $(n-i)$-tuple $s_{\Lambda}$ denotes the coordinates of the second kind with respect to $\bar{X}_{i+1}, \ldots, \bar{X}_{n}$.

Next, observe that since $\tilde{f}_{\Lambda, q} \in S\left(\mathbf{R}^{n+1-i}\right)$ it must be that $\hat{g}_{\Lambda, q}\left(s_{\Lambda}, 0\right)=0$, and by Taylor's formula

$$
\left|\hat{f}_{\Lambda, q}\left(s_{\Lambda}, \xi\right)\right|=\left|\xi\left(\frac{\partial}{\partial \xi}\right) \hat{g}_{\Lambda}\left(s_{\Lambda}, \xi_{0}\right)\right|\left|\xi^{-1}\right|=\left|\hat{Y}_{j} \hat{g}_{\Lambda, q}\left(s_{\Lambda}, \xi_{0}\right)\right|\left|\lambda_{j}\right|^{-1}
$$

for some $\xi_{0}$ between 0 and $\xi$. To estimate the $L^{2}(\Gamma \backslash N)$ norm of $f_{\Lambda, q}$ or, which is the same, the $L^{2}\left(\mathbf{R}^{n+1-i}\right)$ norm $\|\cdot\|$ of $\hat{f}_{\Lambda, q}$ we notice that by (1.4)

$$
\begin{aligned}
\left\|\hat{f}_{\Lambda, q}\right\|^{2} & =\int d s_{\Lambda}\left\{\int_{-1}^{1}+\int_{|\xi| \geq 1}\right\}\left|\hat{g}_{\Lambda, q}\left(s_{\Lambda}, \xi\right)\right|^{2} \xi^{-2} d \xi \\
& \leq \int 2 \sup _{\xi}\left|\hat{Y}_{j} \hat{g}_{\Lambda, q}\left(s_{\Lambda}, \xi\right)\right|^{2} \lambda_{j}^{-2} d s_{\Lambda}+\left\|\hat{g}_{\Lambda, q}\right\|^{2} .
\end{aligned}
$$


Applying the $\mathbf{R}^{1}$-Sobolev inequality

$$
\sup _{\xi}|h(\xi)|^{2} \leq 2\left(\|h\|^{2}+\left\|\frac{d h}{d \xi}\right\|^{2}\right)
$$

to $h(\xi)=\hat{Y}_{j} \hat{g}_{\Lambda, q}\left(s_{\Lambda}, \xi\right)$ in (1.5) and replacing $\partial / \partial \xi$ of (1.6) by $-\lambda_{j}^{-1} \hat{Y}_{j}$ we obtain

$$
\left\|\hat{f}_{\lambda, q}\right\|^{2} \leq 4 \lambda_{j}^{-2}\left(\left\|\hat{Y}_{j} \hat{g}_{\Lambda, q}\right\|^{2}+\lambda_{j}^{-2}\left\|\hat{Y}_{j}^{2} g_{\Lambda, q}\right\|^{2}\right)+\left\|\hat{g}_{\Lambda, q}\right\|^{2} .
$$

To get estimates on $U f_{\Lambda, q}$, for each $U \in \mathcal{U}(\mathfrak{N})$, we begin by writing $\hat{D} \hat{U} \hat{f}_{\Lambda, q}=$ $\hat{U} \hat{g}_{\Lambda, q}+[\hat{D}, \hat{U}] \hat{f}_{\lambda, q}$. Now, if it happens that $[D,[D, U]]=0$, then we substitute $\hat{f}_{\Lambda, q}=\hat{g}_{\Lambda, q} / P_{\Lambda}$ in the right side above and we obtain

$$
\hat{U} \hat{f}_{\Lambda, q}=\left(\hat{U} \hat{g}_{\Lambda, q}+[\hat{D}, \hat{U}] \hat{g}_{\Lambda, q} / P_{\Lambda}\right) / P_{\Lambda}
$$

and so

$$
\hat{U} \hat{f}_{\Lambda, q}=\left(\hat{D} \hat{U} \hat{g}_{\Lambda, q}+[\hat{D}, \hat{U}] \hat{g}_{\Lambda, q}\right) P_{\Lambda}^{-2} .
$$

But, if $(\operatorname{ad} D)^{2} U \neq 0$, then we apply the same reasoning to $[\hat{D}, \hat{U}] \hat{f}_{\Lambda, q}$ as we used previously for $\hat{U} \hat{f}_{\Lambda, q}$. Hence

$$
\hat{U} \hat{f}_{\Lambda, q}=\left(\hat{U} \hat{g}_{\Lambda, q}+\left\{[\hat{D}, \hat{U}] \hat{g}_{\Lambda, q}+[\hat{D}[\hat{D}, \hat{U}]] \hat{f}_{\Lambda, q}\right\} P_{\Lambda}^{-1}\right) P_{\Lambda}^{-1} .
$$

If $(\operatorname{ad} D)^{3} U=0$, then we substitute $\hat{f}_{\Lambda, q}=\hat{g}_{\Lambda, q} / P_{\Lambda}$ and obtain

$$
\hat{U} \hat{f}_{\Lambda, q}=\left(\hat{D}^{2} \hat{U} \hat{g}_{\Lambda, q}+\hat{D}[\hat{D}, \hat{U}] \hat{g}_{\Lambda, q}+[\hat{D}[\hat{D}, \hat{U}]] \hat{g}_{\Lambda, q}\right) P_{\Lambda}^{-3} .
$$

Proceeding inductively, we see that, if $m \geq 1$ is such that $(\operatorname{ad} D)^{m} U=0$, then

$$
\hat{U} \hat{f}_{\Lambda, q}=\hat{h}_{m} P_{\Lambda}^{-m}
$$

where

$$
\begin{aligned}
h_{m}= & {[D[D \cdots[D, U] \cdots]] g_{\Lambda, q}+D[D \cdots[D, U] \cdots] g_{\Lambda, q} } \\
& +\cdots+D^{m-2}[D, U] g_{\Lambda, q}+D^{m-1} U g_{\Lambda, q} \in C^{\infty}(\Gamma \backslash N),
\end{aligned}
$$

and $P_{\Lambda}=i \xi$. Here the first bracket involves $m-1 D$ 's with the number of $D$ 's inside the brackets decreasing by 1 in each successive summand.

Also we notice that if $P_{\Lambda}\left(\xi_{0}\right)=0$ then all the (partial) derivatives of order less than $m$ of the Schwartz function $\hat{h}_{m}=P_{\Lambda}^{m} \hat{U} \hat{f}_{\Lambda, q}$ vanish at $\xi_{0}$. (Of course, here $P_{\Lambda}\left(\xi_{0}\right)=0$ if and only if $\xi_{0}=0$, but later we will refer to this argument in the context of a more general $P_{\Lambda}$.) Hence all the nonzero terms in Taylor's expansion of $\hat{h}_{m}$ at $\xi_{0}$ contain (partial) derivatives of $\hat{h}_{m}$ of order at least $m$ and we have the estimate

$$
\left|\hat{h}_{m}\left(s_{\Lambda}, \xi\right)\right| \leq \sum_{|\alpha|=m}(\alpha !)^{-1} D_{\xi}^{\alpha} \hat{h}_{m}\left(s_{\Lambda}, \xi_{\alpha}\right)||\left(\xi-\xi_{0}\right)^{\alpha} \mid
$$

for suitable $\xi_{\alpha}$.

Thus dividing the above inequality by $P_{\Lambda}^{m}$, for $P_{\Lambda}=i \xi, \xi_{0}=0, \alpha=m$, and $D_{\xi}=-\lambda_{j}^{-1} \hat{Y}_{j}$ we get

$$
\left|\hat{h}_{m} P_{\Lambda}^{-m}\left(s_{\Lambda}, \xi\right)\right| \leq(m !)^{-1} \sup _{\xi}\left|\hat{Y}_{j}^{m} \hat{h}_{m}\left(s_{\Lambda}, \xi\right)\right|\left|\lambda_{j}\right|^{-m} .
$$


Now we esimate the $L^{2}\left(\mathbf{R}^{n-i}\right)$ norm of $\hat{h}_{m} P_{\Lambda}^{-m}$ in exactly the same manner as we estimated $\left\|\hat{f}_{\Lambda, q}\right\|^{2}$ in (1.5) but using now (1.9) instead of (1.4) and we get

$$
\left\|\hat{U} \hat{f}_{\Lambda, q}\right\|^{2} \leq 4(m !)^{-1} \lambda_{j}^{-2 m}\left(\left\|\hat{Y}_{j}^{m} \hat{h}_{m}\right\|^{2}+\lambda_{j}^{-2}\left\|\hat{Y}_{j}^{m+1} \hat{h}_{m}\right\|^{2}\right)+\left\|\hat{h}_{m}\right\|^{2} .
$$

(If $m=1$ and $U=I$ then $\hat{h}_{1}=\hat{g}_{\lambda, q}$ and (1.10) is just (1.7).)

If $X$ has the rational supplmentation property, $\left(1.1^{\prime}\right)$ "eliminates" the $\lambda_{j}$ 's from (1.10). If it has the non-Liouville supplementation, we apply (1.1) to get

$$
\begin{aligned}
\lambda_{j}^{-2 m}\left\|\hat{Y}_{j}^{m} \hat{h}_{m}\right\|^{2} & \leq C^{2}\left\|\left(\Lambda\left(e_{1}\right)^{2}+\cdots+\Lambda\left(e_{n}\right)^{2}\right)^{m d} \hat{Y}_{j}^{m} \hat{h}_{m}\right\|^{2} \\
& =c^{2}\left\|\phi_{j, \Lambda, q}\right\|^{2},
\end{aligned}
$$

and

$$
\begin{aligned}
\lambda_{j}^{-2(m+1)}\left\|\hat{Y}_{j}^{m+1} \hat{h}_{m}\right\|^{2} & \leq C^{2}\left\|\left(\Lambda\left(e_{1}\right)^{2}+\cdots+\Lambda\left(e_{n}\right)^{2}\right)^{(m+1) d} \hat{Y}_{j}^{m+1} \hat{h}_{m}\right\|^{2} \\
& =C^{2}\left\|\hat{\psi}_{j, \Lambda, q}\right\|^{2},
\end{aligned}
$$

where $\phi_{j, \Lambda, q}$ and $\psi_{j, \Lambda, q}$ are defined by the equations above, and where $\phi_{j}=$ $\sum_{\Lambda, q} \phi_{\Lambda, q}$ and $\psi_{j}=\sum_{\Lambda, q} \psi_{\Lambda, q}$ are in $C^{\infty}(\Gamma \backslash N)$. Thus, denoting by \|\|$\cdot \|$ the norm in $L^{2}(\Gamma \backslash N)$, we have (recalling the $j$ which was fixed early in the proof)

$$
\begin{aligned}
\|U f\| \|^{2} & =\sum_{\Lambda, q}\left\|\hat{U} f_{\Lambda, q}\right\|^{2} \\
& \leq \sum_{j=1}^{m} \sum_{\left\{\Lambda, q: \Lambda\left(Z_{j}\right) \neq 0\right\}} C_{1}^{2}\left(\left\|\hat{\phi}_{j, \Lambda, q}\right\|^{2}+\left\|\hat{\psi}_{j, \Lambda, q}\right\|^{2}\right)+2\left\|\hat{h}_{m}\right\|^{2} \\
& \leq \sum_{j} C_{1}^{2}\left(\|\| \phi_{j}\left\|^{2}+\right\|\left\|\psi_{j}\right\|^{2}\right)+2 m\left\|h_{m}\right\| \|^{2},
\end{aligned}
$$

i.e. $f=\sum_{\Lambda, q} f_{\Lambda, q} \in C^{\infty}(\Gamma \backslash N)$ and $D$ is globally regular.

REMARK 1. Suppose $D=X+i Z$ with $X \in \mathfrak{N}$ satisfying the hypothesis of Theorem 1 and $Z \in \mathfrak{Z}$. Then, in the notation of Theorem $1, \hat{D}=i \xi-\Lambda(\bar{Z})$. We could replace (1.3) by

$$
\hat{f}_{\Lambda, q}\left(s_{\Lambda}, \xi\right)=\hat{g}_{\Lambda, q}\left(s_{\Lambda}, \xi\right)(i \xi-\Lambda(\bar{Z}))^{-1},
$$

and then $\left(1.3^{\prime}\right)$ would yield the estimate $\left|\hat{f}_{\Lambda, q}\left(s_{\Lambda}, \xi\right)\right| \leq\left|\hat{g}_{\Lambda, q}\left(s_{\Lambda}, \xi\right)\right||\Lambda(\bar{Z})|^{-1}$ or, more generally, by (1.8), $\left|\hat{U} \hat{f}_{\Lambda, q}\right| \leq\left|\hat{h}_{m}\right||\Lambda(\bar{Z})|^{-m}$, provided $\Lambda(Z) \neq 0$. If now $Z$ were a non-Liouville vector, as in (1.1) we would have

$$
\begin{aligned}
|\Lambda(\bar{Z})|^{-m} & =|\Lambda(Z)|^{-m} \leq C^{m}\left(\Lambda\left(e_{1}\right)^{2}+\cdots+\Lambda\left(e_{n}\right)^{2}\right)^{m d} \\
& =C^{m}\left(\Lambda\left(\bar{e}_{1}\right)^{2}+\cdots+\Lambda\left(\bar{e}_{n}\right)^{2}\right)^{m d}
\end{aligned}
$$

with some rational basis $e_{1}, \ldots, e_{n}$ of $\mathfrak{Z}$. Hence

$$
\|U f\|\left\|^{2} \leq C^{m}\right\|\left\|\left(\Lambda\left(e_{1}\right)^{2}+\cdots+\Lambda\left(e_{n}\right)^{2}\right)^{m d} h_{m}\right\|\left\|^{2}+\sum_{\{\Lambda, q: \Lambda(Z)=0\}}\right\| \hat{U} \hat{f}_{\Lambda, q} \|^{2}
$$

while the sum over those $\Lambda$ such that $\Lambda(Z)=0$, is finite by the estimates of the proof of Theorem 1. What we have just shown can be stated as the following slightly modified version of Theorem 1 . 
THEOREM $1^{\prime}$. Let $N$ be any 1-connected nilpotent Lie group possessing a cocompact discrete subgroup $\Gamma$. If $X \in \mathfrak{N}$ has the non-Liouville supplementation property and $Z \in \mathfrak{Z}(\mathfrak{N})$ (center of $\mathfrak{N}$ ) is a non-Liouville vector then $D=X+i Z$ is globally regular on $\Gamma \backslash N$.

The hypothesis of Theorem $1^{\prime}$ that $Z$ be a non-Liouville vector cannot be relaxed, even if $\mathfrak{N}$ is only two-step, as the following example shows.

EXAMPLE 1. Let $\mathfrak{N}$ be spanned by $\left\{X, Y_{1}, Y_{2}, Z_{1}, Z_{2}\right\}$, where all nontrivial bracket products are generated by $\left[X, Y_{j}\right]=Z_{j}, j=1,2$. Let $D=X+i Z$, where $Z=Z_{1}+\alpha Z_{2}$ with some fixed real number $\alpha$. Let $N=\exp \mathfrak{N}$ and let $\Gamma$ be the discrete cocompact subgroup of $N$ generated by $\exp X, \exp Y_{1}$ and $\exp Y_{2}$. Let $\pi \in(\Gamma \backslash N)^{\wedge}$ be infinite dimensional, and $\Lambda \in O_{N}(\pi)$ with $\Lambda\left(Z_{j}\right)=\lambda_{j} \in \mathbf{Z}$, $j=1,2$. Then there is a Schrödinger model of $\pi$ in $L^{2}(\mathbf{R})$ derived from the Mackey induced representation space using the maximal subordinate subalgebra $\mathfrak{M}$ spanned by $\left\{Y_{1}, Y_{2}, Z_{1}, Z_{2}\right\}$. In this model $\pi(X)=d / d t, \pi\left(Y_{j}\right)=i \lambda_{j} t$, and $\pi\left(Z_{j}\right)=i \lambda_{j}$, $j=1,2$. Taking the (Euclidean) Fourier transform ^ we obtain $\hat{\pi}(X)=i \xi, \hat{\pi}\left(Y_{j}\right)=$ $-\lambda_{j}(\partial / \partial \xi), \hat{\pi}\left(Z_{j}\right)=i \lambda_{j}$, and $\hat{\pi}(D)=i \xi-\left(\lambda_{1}+\alpha \lambda_{2}\right)$. Hence $\hat{f}_{\Lambda}(\xi)=\hat{g}_{\Lambda}(\xi)\left(i \xi-\lambda_{1}-\right.$ $\left.\alpha \lambda_{2}\right)^{-1}$ is the unique Schwartz solution of $\hat{\pi}(D) \hat{f}_{\Lambda}=\hat{g}_{\Lambda}$. If $\alpha$ is a Liouville number, which corresponds to $Z$ being a Liouville vector in the center $\mathfrak{Z}$ of $\mathfrak{N}$, we can find, for each integer $p>0$, a pair $\left(\lambda_{1 p}, \lambda_{2 p}\right)$ such that $\left|\lambda_{1 p}+\alpha \lambda_{2 p}\right|<\left(\left|\lambda_{1 p}\right|+\left|\lambda_{2 p}\right|\right)^{-p}$. Let $\Lambda_{p} \in(\Gamma \backslash N)^{\wedge}$ be such that $\Lambda_{p}\left(Z_{j}\right)=\lambda_{j p}, j=1,2$. Define $g=\sum_{p} g_{p}$ where $g_{p} \in H_{\Lambda p}, p=1,2, \ldots$, in such a way that $\hat{g}_{p}(\xi)=\left(\left|\lambda_{1 p}\right|+\left|\lambda_{2 p}\right|\right)^{-p / 2} \phi(\xi)$ with some fixed $\phi \in C^{\infty}(\mathbf{R}), \phi(\xi)=1$ on a neighborhood of $\xi=0$ and $\phi(\xi)=0$ outside a compact neighborhood of zero. Then $g \in C^{\infty}(\Gamma \backslash N)$, but $\sum_{p}\left|\hat{f}_{p}(0)\right|^{2}$ diverges. By the Sobolev inequality (1.6)

$$
\begin{aligned}
\infty & =\sum_{p}\left|\hat{f}_{p}(0)\right|^{2} \leq \sum_{p} \sup _{\xi}\left|\hat{f}_{p}(\xi)\right|^{2} \leq \sum_{p} 2\left(\left\|\hat{f}_{p}\right\|^{2}+\lambda_{j}^{-2}\left\|\hat{Y}_{j} \hat{f}_{p}\right\|\right) \\
& \leq 2\|\| f\left\|\left.\right|^{2}+\left.2\left|\| Y_{j} f\right|\right|^{2} .\right.
\end{aligned}
$$

Thus $f=\sum_{p} f_{p} \notin C^{\infty}(\Gamma \backslash N)$ and $D$ is not globally regular on $\Gamma \backslash N$.

The hypothesis of Theorem 1 (and also that of Theorem $1^{\prime}$ ) that $X$ has the non-Liouville supplementation property can be replaced by a number theoretic condition, as illustrated by Example 2. The example also shows what obstructions one encounters in attempting to prove a general theorem of this sort.

EXAMPLE 2. Let the Lie algebra $\mathfrak{N}_{n}$ be spanned by the vectors $X, Y_{1}, \ldots, Y_{n-1}$, $Z$, where $Z$ is central. Let the only nontrivial bracket products be generated by $\left[X, Y_{j}\right]=Y_{j+1}, 1 \leq j<n-1$, and $\left[X, Y_{n-1}\right]=Z$. We call $N_{n}=\exp \mathfrak{N}_{n}$ the $n$-step chain group. Let $\Gamma$ be a cocompact discrete subgroup of $N_{n}$ such that $X$ and $Y_{1}$, the generators of $\mathfrak{N}_{n}$, are in $\log \Gamma$. If $\mathfrak{M}$ is spanned by $\left\{Y_{1}, \ldots, Y_{n-1}, Z\right\}$, and if $\Lambda(Z) \neq 0$, then $\mathfrak{M}$ is a $\Gamma$-rational subalgebra of $\mathfrak{N}_{n}$ and is maximal subordinate to $\Lambda$. If we now let $n=3$, and $D=Y_{1}+\beta Y_{2}+\gamma Z(\in \mathfrak{M})$, we will show that $D$ is globally regular on $\Gamma \backslash N_{3}$ if $\beta^{2} / 2-\gamma$ is not a Liouville number. (In particular, if both $\beta$ and $\gamma$ are algebraic numbers, then $\beta^{2} / 2-\gamma$ is non-Liouville.) Here $\left[D, \mathfrak{N}_{1}\right]=\{0\} \varsubsetneqq \mathbf{R} Z=\mathfrak{N}_{2}$, and consequently $D$ does not have any form of the supplementation property. 
All the nonequivalent unitary irreducible representations in the spectrum of $\Gamma \backslash N_{3}$ which are nontrivial on the center can be parametrized by pairs of integers $(a, c)$ with $a \neq 0$ that correspond to integral $\Lambda \in \mathfrak{N}_{3}^{*} / \operatorname{Ad} N_{3}$ with $\Lambda(Z)=a$, $\Lambda\left(Y_{1}\right)=c, \Lambda\left(Y_{2}\right)=0$. All those representations, say $\pi_{a c}$, can be realized on $L^{2}(\mathbf{R})$ in such a way that (cf. $\left[\mathbf{4}\right.$, p. 119]) $\pi_{a c}(X)=d / d x, \pi_{a c}\left(Y_{1}\right)=2 \pi i\left(c+a x^{2} / 2\right)$, $\pi_{a c}\left(Y_{2}\right)=2 \pi i a x$, and $\pi_{a c}(Z)=2 \pi i a$. Thus

$$
\left.\pi_{a c}(D)=2 \pi i\left(c+a x^{2} / 2\right)+\beta a x+\gamma a\right]=a \pi i\left(x-x_{+}\right)\left(x-x_{-}\right),
$$

with $x_{ \pm}=-\beta \pm\left(\beta^{2}-2 \gamma-2 c / a\right)^{1 / 2}$. Therefore

$$
\tilde{f}_{a c}(x)=\tilde{g}_{a c}(x)\left[\pi i a\left(x-x_{+}\right)\left(x-x_{-}\right)\right]^{-1}
$$

is the unique Schwartz solution of the equation $\pi_{a c}(D) \tilde{f}_{a c}=\tilde{g}_{a c}$ and we can write

$$
\begin{aligned}
\tilde{f}_{a c}(x)=\left[\pi i a\left(x_{+}-x_{-}\right)\right]^{-1}\left[\tilde{g}_{a c}(x)\left(x-x_{+}\right)^{-1}-\tilde{g}_{a c}(x)\left(x-x_{-}\right)^{-1}\right], \\
\text { if } x_{+}-x_{-}=2\left(\beta^{2}-2 \gamma-2 c / a\right)^{1 / 2} \neq 0,
\end{aligned}
$$

or

$$
\tilde{f}_{a c}(x)=\tilde{g}_{a c}(x)(x-\beta)^{2} / \pi i a \quad \text { otherwise. }
$$

Now, if $\beta^{2} / 2-\gamma$ is not a Liouville number, there exist a positive constant $K$ and a positive integer $p$ such that $\left|\beta^{2}-2 \gamma-2 c / a\right| \geq K^{-2}(2 \pi a)^{-2(p+1)}$ for all integers $c$ and $a, a \neq 0$. Hence

$$
\begin{aligned}
\left|\tilde{f}_{a c}(x)\right| & \leq K\left|\tilde{g}_{a c}(x)(2 \pi a)^{p} /\left(x-x_{+}\right)\right|+K\left|\tilde{g}_{a c}(x)(2 \pi a)^{p} /\left(x-x_{-}\right)\right| \\
& =K\left|\tilde{Z}^{p} \tilde{g}_{a c}(x)\right| /\left|x-x_{+}\right|+K\left|\tilde{Z}^{p} \tilde{g}_{a c}(x)\right| /\left|x-x_{-}\right| .
\end{aligned}
$$

If $x_{+}$and $x_{-}$are complex roots, then for all real $x$,

$$
\left|x-x_{ \pm}\right| \geq\left(\beta^{2}-2 \gamma-2 c / a\right)^{1 / 2} \geq K^{-1}(2 \pi a)^{-p-1},
$$

and we have the estimate $\left|\tilde{Z}^{p} \tilde{g}_{a c}(x)\right| /\left|x-x_{ \pm}\right| \leq K\left|\tilde{Z}^{2 p+1} \tilde{g}_{a c}(x)\right|$ which implies the corresponding norm estimate $\left\|\tilde{Z}^{p} \tilde{g}_{a c} /\left(x-x_{ \pm}\right)\right\| \leq K\left\|\tilde{Z}^{2 p+1} \tilde{g}_{a c}\right\|$. If $x_{+}$and $x_{-}$ are real, we notice that $\tilde{g}_{a c}\left(x_{ \pm}\right)=0$ and we use Taylor's expansion of $\tilde{Z}^{p} \tilde{g}_{a c}(x)$ at $x=x_{ \pm}$to get to estimate

$$
\left|\tilde{Z}^{p} \tilde{g}_{a c}(x)\right| /\left|x-x_{ \pm}\right| \leq \sup _{x}\left|\tilde{X} \tilde{Z}^{p} \tilde{g}_{a c}(x)\right|
$$

To estimate $\left\|\tilde{f}_{\text {ac }}\right\|$, as in (1.5) we break the integral $\int \cdots d x$ over $\mathbf{R}$ into two integrals: one over the interval $\left|x-x_{ \pm}\right| \leq 1$ and the other one over its complement $\left|x-x_{ \pm}\right|>$ 1 , we apply (1.12) to the integrand of the first integral and then use Sobolev's inequality (1.6) with $h(x)=\tilde{X} \tilde{Z}^{p} \tilde{g}_{a c}(x)$. The resulting estimate is

$$
\left\|\tilde{f}_{a c}\right\|^{2} \leq 8 K^{2}\left(\left\|\tilde{X} \tilde{Z}^{p} \tilde{g}_{a c}\right\|^{2}+\left\|\tilde{X}^{2} \tilde{Z}^{p} \tilde{g}_{a c}\right\|^{2}\right)+2 K^{2}\left\|\tilde{Z}^{p} \tilde{g}_{a c}\right\|^{2}
$$

Next we proceed as we did in (1.8) to obtain the estimates on $\tilde{U} \tilde{f}_{a c}, U \in \mathcal{U}\left(\mathfrak{N}_{3}\right), a$, $c$ integers, $a \neq 0$. The remaining $\infty$-dimensional representations of $N_{3}$ which are in the spectrum of $\Gamma \backslash N_{3}$ correspond to integral $\Lambda \in \mathfrak{N}_{3}^{*} / \operatorname{Ad} N_{3}$ which are 0 on the center $\mathbf{R} Z$ of $\mathfrak{N}_{3}$, and therefore can be viewed as representations of the quotient group $\bar{N}_{3}=N_{3} / \exp \mathbf{R} Z$ isomorphic to the 3-dimensional Heisenberg group. On this quotient group $\bar{N}_{3}, \bar{D}=\bar{Y}_{1}+\beta \bar{Y}_{2}$ already has the rational supplementation property: $\left[\bar{D}, \overline{\mathfrak{N}}_{3}\right]=\mathbf{R} \bar{Y}_{2}$, and is thus globally regular by Theorem 1 . 
Conversely, if $\beta^{2} / 2-\gamma$ is a Liouville number that can be rapidly approximated by rationals from the right, i.e. if there exists an infinite sequence of rational numbers $p_{n} / q_{n},\left(p_{n}, q_{n}\right)=1$, such that $0<p_{n} / q_{n}-\beta^{2} / 2+\gamma<q_{n}^{-n}$, then $D=Y_{1}+\beta Y_{2}+\gamma Z$ is not globally regular. (Clearly there exist such Liouville numbers; that they do not comprise the whole set of Liouville numbers follows from the properties of convergents of continued fractions.) To show this we may actually assume that there is a sequence $\left\{p_{n} / q_{n}\right\}$ of rational numbers such that $0<p_{n} / q_{n}-\beta^{2} / 2+\gamma<$ $\left(q_{n}+\left|p_{n}\right|\right)^{-n}$. Let $g=\sum_{n=1}^{\infty} g_{n} \in C^{\infty}(\Gamma \backslash N)$ be such that each $g_{n}$ is in an (irreducible) representation space $H_{\pi} \subset L^{2}(\Gamma \backslash N)$ of $\pi_{q_{n} p_{n}}$ and corresponds to $\tilde{g}_{n}(x)=\left(q_{n}+\left|p_{n}\right|\right)^{-n / 2} \psi(x)$ in the $L^{2}(\mathbf{R})$ realization of $H_{\pi}$, as described above $\left(\psi(x) \in C^{\infty}(\mathbf{R}), \psi(-\beta)=1, \psi \geq 0\right.$, compactly supported). We have the following estimates on the solutions $\tilde{f}_{n}$ of $\pi_{q_{n} p_{n}}(D) \tilde{f}_{n}=\tilde{g}_{n}$ :

$$
\begin{aligned}
\sup \left|\tilde{f}_{n}(x)\right| & =\sup \left|\tilde{g}_{n}(x) /\left[\pi i q_{n}\left(x-x_{+}\right)\left(x-x_{-}\right)\right]\right| \\
& \geq\left|\tilde{g}_{n}(-\beta) / \pi q_{n}\left(\beta^{2}-2 \gamma-2 p_{n} / q_{n}\right)\right| \geq\left(q_{n}+\left|p_{n}\right|\right)^{n-n / 2-1}
\end{aligned}
$$

where $x_{ \pm}=-\beta \pm i\left(p_{n} / q_{n}-\beta^{2}+2 \gamma\right)^{1 / 2}$, and the sup is taken over all $x \in \mathbf{R}$. As in Example 1, this shows that $f=\sum f_{n} \notin C^{\infty}(\Gamma \backslash N)$. (Note that the right sidedness of the Liouville number was used to insure that neither $x_{+}$nor $x_{-}$is real.)

On the chain group $N_{n}$, any $D$ without the supplementation property acts in the representation space of $\pi_{\Lambda}$ realized on $L^{2}(\mathbf{R})$ as a multiplication by a polynomial, say $P_{\Lambda}(x)$. Example 2 exhibits that the crucial thing for the global regularity of such $D$ then is how rapidly the distances from the real line of the complex roots of $P_{\Lambda}(x)$ vary with $\Lambda \in \mathfrak{N}_{3}^{*} / \operatorname{Ad}^{*} N_{3}$ "as $\Lambda \rightarrow \infty$ ". We will finish this section with the following

THEOREM 2. Let $N$ be any 1-connected nilpotent Lie group possessing a cocompact discrete subgroup $\Gamma$. Let $D=Y \in \mathfrak{N}_{k-1}$-the next to the last step of the lower central series of $\mathfrak{N}$, the Lie algebra of $N$. Suppose $Y$ is a non-Liouville vector (relative to $\Gamma$ ). Then $D$ is globally regular on $\Gamma \backslash N$.

Proof. The hypothesis in Theorem 1 that $X$ has supplementation was to assure the existence of $\bar{Y}_{j}$ in $\overline{\mathfrak{N}}_{k-1}$ such that $\left[\bar{X}, \bar{Y}_{j}\right]=\bar{Z}_{j}$, so that we could build a Schrödinger model for the representation $\pi_{\Lambda}$ in which $\pi_{\Lambda}(\bar{X})=\partial / \partial x$, $\pi_{\Lambda}\left(\bar{Y}_{j}\right)=i \Lambda\left(\bar{Z}_{j}\right) x$, and $\pi_{\Lambda}\left(\bar{Z}_{j}\right)=i \Lambda\left(Z_{j}\right)$. We can now reverse the roles of $X$ and $Y_{j}$ in this picture. That is, we start with $D=Y \in \mathfrak{N}_{k-1}$ and a rational basis $Z_{1}, \ldots, Z_{n}$ of $\mathfrak{N}_{k}$. Then for given $\Lambda$, if $\Lambda\left(Z_{j}\right) \neq 0$, either $\bar{Y}$ is in the center of $\overline{\mathfrak{N}}$ (a quotient of $\mathfrak{N}$ with one-dimensional center spanned by $\bar{Z}_{j}$ ), in which case we get the estimates on $\pi_{\Lambda}(\bar{D})$ using (1.1) as we did in (1.11), with $m=1$, and noting that the passage from $\mathfrak{N}$ to $\overline{\mathfrak{N}}$ does not affect these estimates, or else $\bar{Y} \in \overline{\mathfrak{N}}_{k-1} \sim$ center of $\overline{\mathfrak{N}}$. It is in the former case that we need non-Liouville, while in the latter case, there exists $\bar{X}_{j} \in \overline{\mathfrak{N}}$ such that $\left[\bar{X}_{j}, \bar{Y}\right]=\bar{Z}_{j}$. We again build a Schrödinger model for $\pi_{\Lambda}$ in which $\pi_{\Lambda}\left(\bar{X}_{j}\right)=\partial / \partial x, \pi_{\Lambda}(\bar{Y})=i \Lambda\left(Z_{j}\right) x$, and $\pi_{\Lambda}\left(\bar{Z}_{j}\right)=i \Lambda\left(\bar{Z}_{j}\right)$. Again, $\tilde{f}_{\Lambda}(x)=\tilde{g}_{\Lambda}(x)\left[i \Lambda\left(Z_{j}\right) x\right]^{-1}$ is the only Schwartz solution of $\pi_{\Lambda}(D) \tilde{D}_{\Lambda}=\tilde{g}_{\Lambda}$, and we can use $\pi_{\Lambda}\left(X_{j}\right)=\partial / \partial x$ in Taylor's formula and Sobolev's inequality that are needed for the estimates on $\tilde{U} \tilde{f}_{\Lambda}, U \in \mathcal{U}(\mathfrak{N})$, as in the proof of Theorem 1 , noticing that $\left|\Lambda\left(Z_{j}\right)\right|^{-1} \leq M_{j}$ for all integral on $\mathfrak{M}$ such that $\Lambda\left(Z_{j}\right) \neq 0$.

We mention also the following modification of Theorem 2 . 
THEOREM $2^{\prime}$. Let $N$ be any 1-connected nilpotent Lie group possessing a cocompact discrete subgroup $\Gamma$. Let $D=Y+i Z$ with $Y \in \mathfrak{N}_{k-1}$ and $Z \in$ the center of $\mathfrak{N}$. Suppose that both $Y$ and $Z$ are non-Liouville vectors (relative to $\Gamma$ ). Then $D$ is globally regular on $\Gamma \backslash N$.

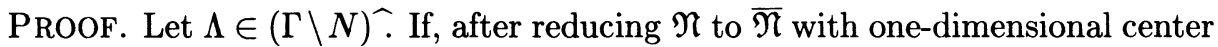
$\overline{\mathfrak{Z}}, \bar{Y} \in \overline{\mathfrak{Z}}$, then $\pi_{\Lambda}(D)=i \Lambda(Y)-\Lambda(Z), \tilde{f}_{\Lambda}=\tilde{g}_{\Lambda}(i \Lambda(Y)-\Lambda(Z))^{-1}$, and we use either the estimate $\left|\tilde{f}_{\Lambda}\right| \leq\left|\tilde{g}_{\Lambda}\right||\Lambda(Z)|^{-1}$ if $\Lambda(Z) \neq 0$, or we use $\left|\tilde{f}_{\Lambda}\right| \leq\left|\tilde{g}_{\Lambda}\right||\Lambda(Y)|^{-1}$ if $\Lambda(Z)=0$, and the proof goes now as in Theorem $1^{\prime}$ using (1.1). If, on the other hand, $\bar{Y} \notin \overline{\mathfrak{Z}}$, then $\pi_{\Lambda}(D)=i \Lambda\left(Z_{j}\right) x-\Lambda(Z)$, again we proceed as in the proof of Theorem $1^{\prime}$ when $\Lambda(Z) \neq 0$, while if $\Lambda(Z)=0$, we are in the situation of Theorem 2 ; cf. the comment at the beginning of the proof of Theorem $1, \S 1$.

2. n-step nilmanifolds - complex fields. We can "complexify" Theorem 2 as follows. Suppose $D=Y_{1}+i Y_{2}$ with $Y_{1}, Y_{2} \in \mathfrak{N}_{k-1}$, both non-Liouville vectors. If $\mathfrak{N}$ is of step 3 or more, then $\left[Y_{1}, Y_{2}\right]=0$. If $\mathfrak{N}$ is of step $2, \mathfrak{N}_{k-1}=\mathfrak{N}$, and we simply assume that $\left[Y_{1}, Y_{2}\right]=0$. Let $Z_{1}, \ldots, Z_{n}$ be a fixed rational basis of $\mathfrak{N}_{k}$, $\Lambda \in \mathfrak{N}^{*}$, rational (as remarked after Theorem 0 ), $\Lambda\left(Z_{j}\right) \neq 0$ for some $j$, be given. After reducing to one-dimensional center, we may assume that $\bar{Y}_{1}, \bar{Y}_{2} \in \overline{\mathfrak{N}}_{k-1} \sim$ $\overline{\mathfrak{Z}}$. (Otherwise we would use the estimates from the proof of Theorem $2^{\prime}$, noting that these estimates are independent of the passage from $N$ to $\bar{N}$.) Therefore, there exists $X_{j} \in \mathfrak{N}$ such that $\left[\bar{X}_{j}, \bar{Y}_{1}\right]=\bar{Z}_{j}$. We construct a Schrödinger model for $\pi_{\Lambda}$ choosing $X_{j}$ to be the first external vector. Since $Y_{2} \in \mathfrak{N}_{k-1}$, we have $\left[\bar{X}_{j}, \bar{Y}_{2}\right] \in \mathfrak{N}_{k} \subseteq \operatorname{center}(\mathfrak{N})=\mathbf{R} \bar{Z}_{j}$, so $\left[\bar{X}_{j}, \bar{Y}_{2}\right]=\alpha_{j} \bar{Z}_{j}$ for some $\alpha_{j} \in \mathbf{R}$. If $\bar{Y}_{2} \in$ center of $\mathfrak{Z}\left(\bar{Y}_{1}\right)$, then in our model

$$
\begin{aligned}
& \pi_{\Lambda}\left(Y_{1}\right)=i \Lambda\left(Z_{j}\right) x, \quad \pi_{\Lambda}\left(X_{j}\right)=\frac{\partial}{\partial x} \\
& \pi_{\Lambda}\left(Y_{2}\right)=i \Lambda\left(\left[X_{j}, Y_{2}\right]\right) x, \quad \text { and } \quad \pi_{\Lambda}\left(Z_{j}\right)=i \Lambda\left(Z_{j}\right) .
\end{aligned}
$$

Thus $\pi_{\Lambda}(D)=\left\{i \Lambda\left(Z_{j}\right)-\Lambda\left(\left[X_{j}, Y_{2}\right]\right)\right\} x$ and we can use the same estimates on $\tilde{f}_{\Lambda}$, unique Schwartz solution of $\pi_{\Lambda}(D) \tilde{f}_{\Lambda}=\tilde{g}_{\Lambda}$, as we did in the proof of Theorem 2 (we notice that $\left|i \Lambda\left(Z_{j}\right)-\Lambda\left(\left[X_{j}, Y_{2}\right]\right)\right|^{-1} \leq\left|\Lambda\left(Z_{j}\right)\right|^{-1}$ ). If on the other hand, $\bar{Y}_{2} \notin$ center of $\mathfrak{Z}\left(\bar{Y}_{1}\right)$, then there exists $\bar{V}_{j} \in \mathfrak{Z}\left(\bar{Y}_{1}\right)$ such that $\left[\bar{V}_{j}, \bar{Y}_{2}\right]=\bar{Z}_{j}$ in $3\left(\bar{Y}_{1}\right)$, and we pick $V_{j}$ to be the second external vector in our model. In this model $\pi_{\Lambda}\left(Y_{1}\right)=i \Lambda\left(Z_{j}\right) x$

$$
\pi_{\Lambda}\left(X_{j}\right)=\frac{\partial}{\partial x}, \quad \pi_{\Lambda}\left(Y_{2}\right)=i \Lambda\left(Z_{j}\right) v+i \Lambda\left(\left[X_{j}, Y_{2}\right]\right) x
$$

and

$$
\pi_{\Lambda}\left(V_{j}\right)=\frac{\partial}{\partial v}+i \Lambda\left(\left[X_{j}, V_{j}\right]\right) x
$$

provided $\left[X_{j}, V_{j}\right]$ is central. Hence

$$
\pi_{\Lambda}(D)=i \Lambda\left(Z_{j}\right) x-\left\{\Lambda\left(Z_{j}\right) v+\Lambda\left(\left[X_{j}, Y_{2}\right]\right) x\right\} \equiv P_{\Lambda}(x, v),
$$

and $\left|P_{\Lambda}(x, v)\right|^{2}=\langle A(x, v),(x, v)\rangle$, where the real symmetric matrix $A=\left(\begin{array}{ll}a & b \\ b & c\end{array}\right)$, and $a=\Lambda\left(Z_{j}\right)^{2}+\Lambda\left(\left[X_{j}, Y_{2}\right]\right)^{2}, b=\Lambda\left(Z_{j}\right) \Lambda\left(\left[X_{j}, Y_{2}\right]\right)$, and $c=\Lambda\left(Z_{j}\right)^{2}$. Then the 
minimum eigenvalue of $A$ is positive and is readily calculated to be

$$
\begin{aligned}
c_{\Lambda j}=2\left(a c-b^{2}\right)\left\{a+c+\left((a-c)^{2}+4 b^{2}\right)^{1 / 2}\right\}^{-1} \\
=2 \Lambda\left(Z_{j}\right)^{4}\left\{2 \Lambda\left(Z_{j}\right)^{2}+\Lambda\left(\left[X_{j}, Y_{2}\right]\right)^{2}\right. \\
\left.\quad+\left|\Lambda\left(\left[X_{j}, Y_{2}\right]\right)\right|\left(\Lambda\left(\left[X_{j}, Y_{2}\right]\right)^{2}+4 \Lambda\left(Z_{j}\right)^{2}\right)^{1 / 2}\right\}^{-1} .
\end{aligned}
$$

Then

$$
\left|P_{\Lambda}(x, v)\right|^{2} \geq c_{\Lambda j}\left(x^{2}+v^{2}\right), \quad \text { for all real } x \text { and } v .
$$

In order to obtain $L^{2}$-estimates on $\tilde{f}_{\Lambda}$, the unique Schwartz solution of $\pi_{\Lambda}(D) \tilde{f}_{\Lambda}=$ $\tilde{g}_{\Lambda}$, we proceed as in the proof of Theorem 1, with small modifications. First, $P_{\Lambda}(x, v)=0$ iff $x=v=0$ implies that $\tilde{g}_{\Lambda}\left(s_{\Lambda}, 0,0\right)=0$. Instead of (1.4) we now apply Taylor's formula on $\mathbf{R}^{2}$ combined with the expressions (2.2) for the partial derivatives $\partial / \partial x$ and $\partial / \partial v$. Thus by elementary inequalities we have

$$
\begin{aligned}
& \left|\tilde{f}_{\Lambda, q}\left(s_{\Lambda}, v, x\right)\right|^{2} \leq\left(\left|x\left(\frac{\partial \tilde{g}_{\Lambda}}{\partial x}\right)\left(s_{\Lambda}, v_{0}, x_{0}\right)\right|+\left|v\left(\frac{\partial \tilde{g}_{\Lambda}}{\partial v}\right)\left(s_{\Lambda}, v_{1}, x_{1}\right)\right|\right)^{2} \cdot P_{\Lambda}(v, x)^{-2} \\
& \quad \leq c_{\Lambda j}^{-1}\left(\sup \left|\tilde{X}_{j} \tilde{g}_{\Lambda}\left(s_{\Lambda}, v, x\right)\right|^{2}+\sup \left|\left(\tilde{V}_{j}+i\left[\tilde{X}_{j}, \tilde{V}_{j}\right] \tilde{Y}_{1} \Lambda\left(Z_{j}\right)^{-1}\right) \tilde{g}_{\Lambda}\left(s_{\Lambda}, v, x\right)\right|^{2}\right) \\
& \quad \leq c_{\Lambda j}^{-1}\left(\sup \left|\tilde{X}_{j} \tilde{g}_{\Lambda}\right|^{2}+2 \sup \left|\tilde{V}_{j} \tilde{g}_{\Lambda}\right|^{2}+2 \sup \left|\left[\tilde{X}_{j}, \tilde{V}_{j}\right] \tilde{Y}_{1} \tilde{g}_{\Lambda}\right|^{2} M_{j}^{2}\right)
\end{aligned}
$$

where $M_{j}=\operatorname{Sup}\left\{\left|\Lambda\left(Z_{j}\right)\right|^{-1}: \Lambda \in(\Gamma \backslash N)^{\hat{T}}, \Lambda\left(Z_{j}\right) \neq 0\right\}$ as in $\left(1.1^{\prime}\right)$, and all the suprema are taken over $v, x \in \mathbf{R}$. We notice that for any Schwartz $h_{\Lambda}$, by (2.3), we have

$$
\begin{aligned}
c_{\Lambda j}^{-1}\left|\tilde{h}_{\Lambda}\right|^{2} & \leq \frac{1}{2} M_{j}^{4}\left\{2 \Lambda\left(Z_{j}\right)^{2}+\Lambda\left(\left[X_{j}, Y_{2}\right]\right)^{2}+2\left|\Lambda\left(\left[X_{j}, Y_{2}\right]\right) \Lambda\left(Z_{j}\right)\right|\right\}\left|\tilde{h}_{\Lambda}\right|^{2} \\
& \leq \frac{3}{2} M_{j}^{4}\left(\left|\tilde{Z}_{j} \tilde{h}_{\Lambda}\right|^{2}+\left|\left[\tilde{X}_{j}, \tilde{Y}_{2}\right] \tilde{h}_{\Lambda}\right|^{2}\right) .
\end{aligned}
$$

Applying this inequality to $\tilde{h}_{\Lambda}$ equal subsequently to $\tilde{X}_{j} \tilde{g}_{\Lambda}, \tilde{V}_{j} \tilde{g}_{\Lambda}$, and $\left[\tilde{X}_{j} \tilde{V}_{j} \mid \tilde{Y}_{1} \tilde{g}_{\Lambda}\right.$, we get from (2.4)

$$
\begin{aligned}
& \left|\tilde{f}_{\Lambda, q}\left(s_{\Lambda}, v, x\right)\right|^{2} \\
& \quad \leq \frac{3}{2} M_{j}^{4} \sum_{k=1}^{2}\left(\sup \left|\tilde{U}_{j k} \tilde{X}_{j} \tilde{g}_{\Lambda}\right|^{2}+2 \sup \left|\tilde{U}_{j k} \tilde{V}_{j} \tilde{g}_{\Lambda}\right|^{2}+2 \sup \left|\tilde{U}_{j k}\left[\tilde{X}_{j}, \tilde{V}_{j}\right] \tilde{Y}_{1} \tilde{g}_{\Lambda}\right|^{2} M_{j}^{2}\right)
\end{aligned}
$$

with $U_{j 1}=Z_{j}$ and $U_{j 2}=\left[X_{j}, Y_{2}\right]$. Next, instead of $\int \cdots d \xi$ in (1.5) we use $\iint \cdots d v d x$ and then we break this double integral into two integrals, one over the unit disk $x^{2}+v^{2} \leq 1$ and the other one over its complement, $x^{2}+v^{2} \geq 1$. (Here we are using (2.3).) And finally, instead of (1.6) we use the $\mathbf{R}^{2}$-Sobolev inequality

$$
\sup |f(v, x)|^{2} \leq C\left(\|f\|^{2}+\left\|\frac{\partial^{2} f}{\partial \xi^{2}}\right\|^{2}+\left\|\frac{\partial^{2} f}{\partial \eta^{2}}\right\|^{2}\right)
$$

which we apply to each of the summands in (2.4). (We note that $\left|c_{\Lambda j}\right|^{-1}$ grows at most polynomially in $\Lambda$.) For the estimates on $\tilde{U} \tilde{f}_{\Lambda, q}, U \in \mathcal{U}(\mathfrak{N})$, we proceed as in (1.8) with the same modifications as described above. All this procedure works fine, except that for a general $\mathfrak{N}$ it is hard to tell whether we can actually pick $X_{j}$ and $V_{j}$ so that $\left[X_{j}, V_{j}\right]$ needed in $(2.1)$ is central. It surely is central if $\mathfrak{N}$ is of step two, and we have the following proposition. 
Proposition 1. Let $N$ be a simply connected step 2 nilpotent Lie group, and $\Gamma$ a cocompact discrete subgroup of $N$. If $X, Y \in \mathfrak{N}$ commute, and both are nonLiouville vectors, then $D=X+i Y$ is globally regular on $\Gamma \backslash N$.

Another natural class of groups for which $\left[X_{j}, V_{j}\right]=0$ is that of nilpotent semidirect products of Abelian groups (metabelian groups). For let $\mathfrak{N}=\mathfrak{A} \ltimes \mathfrak{B}$ with $\mathfrak{A} \simeq \mathbf{R}^{p}, \mathfrak{B} \simeq \mathbf{R}^{q}$. Then, if the above constructed $X_{j}$ and $V_{j}$ are such that $X_{j}=\xi_{1}+\eta_{1}$ and $V_{j}=\xi_{2}+\eta_{2}$ with some $\xi_{i} \in \mathfrak{A}, \eta_{i} \in \mathfrak{B}, i=1,2$, we can simply replace them by $X_{j}^{\prime}=\xi_{1}$ and $V_{j}^{\prime}=\xi_{2}$. We summarize these facts as the following

THEOREM $2_{\mathbf{C}}$. Let $N$ be a metabelian nilpotent Lie group with a cocompact discrete subgroup $\Gamma$. Let $D=Y_{1}+i Y_{2}$ with $Y_{1}, Y_{2} \in \mathfrak{N}_{k-1}$-the next to the last step of the lower central series of $\mathfrak{N}$, the Lie algebra of $N$. Suppose both $Y_{1}$ and $Y_{2}$ are non-Liouville vectors (relative to $\Gamma$ ). Then $D$ is globally regular on $\Gamma \backslash N$. If $N$ is of step less than 3 assume moreover that $Y_{1}$ and $Y_{2}$ commute.

Of course $X_{j}$ and $V_{j}$ may commute even if $\mathfrak{N}$ is not metabelian.

EXAMPLE 1. Let $\mathfrak{N}$ be the nilpotent Lie algebra of strictly upper triangular $n \times n$ real matrices, $n \geq 4$. Then $\mathfrak{N}$ is of step $n-1$ and the next to the last stage of $\mathfrak{N}$ is spanned by the two vectors $E_{1, n-1}$ and $E_{2, n}\left(E_{k l}\right.$ denotes the matrix which has 1 on the crossing of $k$ th row with $l$ th column and zeros otherwise) and the one-dimensional center by $E_{n n} . D=E_{1, n-1}+i E_{2, n}$ is globally regular on any nilmanifold $\Gamma \backslash \exp \mathfrak{N}$ such that $E_{1, n-1}, E_{2, n}$ are non-Liouville with respect to the $\Gamma$ rational basis. The (commuting) external vectors $X_{1}$ and $V_{1}$ can be chosen to be $-E_{n-1, n}$ and $E_{1,2}$ respectively.

This covers (infinite-dimensional $\pi$ corresponding to) $\Lambda$ in general position: $\Lambda\left(E_{1 n}\right) \neq 0$. However there are also exceptional orbits with $\operatorname{dim} \pi=\infty$. For these, $\Lambda\left(E_{1 n}\right)=0$ and passage to the quotient puts $\bar{E}_{1, n-1}$ and $\bar{E}_{2, n}$ in the center of $\overline{\mathfrak{N}}$, and so the required Sobolev estimates for such $\Lambda$ are easier, and do not require a choice of $V_{j}$.

We will now quote two examples of 3 -step nilpotent metabelian groups which will also be useful to illustrate our next theorems.

EXAMPLE 2. $\mathfrak{N}=\mathfrak{A} \ltimes \mathfrak{B}$ where $\mathfrak{A}=\mathbf{R} X_{1} \oplus \mathbf{R} X_{2}, \mathfrak{B}=\mathbf{R}$-span of $\left\{U_{1}, U_{2}, U_{12}\right.$, $\left.Y_{1}, Y_{2}, Z\right\}$ with all nontrivial bracket products generated by $\left[X_{j}, U_{j}\right]=Y_{j},\left[X_{j}, Y_{j}\right]$ $=Z$, and $\left[X_{j}, U_{12}\right]=Y_{3-j}, j=1,2[\mathbf{1 7}]$. Here $\mathfrak{N}_{1}=[\mathfrak{N}, \mathfrak{N}]=\mathbf{R} Y_{1} \oplus \mathbf{R} Y_{2} \oplus \mathbf{R} Z$ is the next to the last stage of the lower central series and $N=\exp \mathfrak{N}$.

EXAMPLE 3. $\mathfrak{N}=$ subalgebra of $\mathfrak{N}$ of Example 2 that does not contain $U_{12}$, and $N=\exp \mathfrak{N}$.

In each of these two examples $D=Y_{1}+i Y_{2}$ is globally regular, the commuting external vectors being $X_{1}$ and $X_{2}$, if $Y_{1}$ and $Y_{2}$ are non-Liouville with respect to some $\Gamma$-rational basis.

REMARK. We notice that the model we just built to prove Theorem $2_{\mathbf{C}}$, shows that for $D=X_{j}+i V_{j}, j$-fixed, $\left[X_{j}, V_{j}\right]=0$, we can produce estimates similar to the ones we obtained for $D=Y_{1}+i Y_{2}$. To see this we apply the (Euclidean) Fourier transform to $(2.2)$ obtaining

$$
\begin{aligned}
& \hat{\pi}_{\Lambda}\left(X_{j}\right)=i \xi, \quad \hat{\pi}_{\Lambda}\left(Y_{1}\right)=-\Lambda\left(Z_{j}\right) \frac{\partial}{\partial \xi}, \quad \hat{\pi}_{\Lambda}\left(V_{j}\right)=i \eta \\
& \hat{\pi}_{\Lambda}\left(Y_{2}\right)=-\Lambda\left(Z_{j}\right) \frac{\partial}{\partial \eta}-\Lambda\left(\left[X_{j}, Y_{2}\right]\right) \frac{\partial}{\partial \xi}, \quad \text { and } \quad \hat{\pi}_{\Lambda}(D)=i \xi-\eta
\end{aligned}
$$


Now we can repeat the argument of Theorem $2_{\mathbf{C}}$ using $\partial / \partial \xi=-\Lambda\left(Z_{j}\right)^{-1} \hat{Y}_{1}$ and $\partial / \partial \eta=-\Lambda\left(Z_{j}\right)^{-1}\left(Y_{2}-Y_{1}\left[X_{j}, Y_{2}\right] \Lambda\left(Z_{j}\right)^{-1}\right)$. In particular we have sufficient $L^{2}$-estimates on $f_{\Lambda}$ for the global regularity of $D=X_{1}+i V_{1}$ in Example 3, and in Examples 4 and 5 to follow, provided $\Lambda(Z) \neq 0$ there.

Now we would like to determine the algebraic conditions on (commuting) vector fields $X, V \in \mathfrak{N}$ that would guarantee the existence of a Schrödinger model for $\pi_{\Lambda}$ satisfying (2.1), which would in turn, as described above, imply global regularity of $D=X+i V$. To make $X$ the first external vector in our model, as in the proof of Theorem 1 , we pick $Y_{j} \in \mathfrak{N}_{k-1}$, and a non-Liouville $Z_{j} \in \mathfrak{N}_{k}$ such that $\left[X, Y_{j}\right]=Z_{j}$ assuming therefore that $X$ has the non-Liouville supplementation property. Next, to use $V$ as a second external vector we need two things: $\left(1^{\circ}\right) \bar{V}$ to be in the centralizer of $\bar{Y}_{j}$ in $\overline{\mathfrak{N}}$, and $\left(2^{\circ}\right) \bar{V}$ to bracket a vector, say $Y_{j}^{\prime}$, in the next to the last step of the lower central series of $3\left(\bar{Y}_{1}\right)$, or of $\overline{\mathfrak{N}}$ in particular, onto $\bar{Z}_{j}$. The following condition - a property of a pair of vectors $X$ and $V$, stronger than the requirement that both $X$ and $V$ have the non-Liouville supplementation property-is sufficient for $1^{\circ}$ and $2^{\circ}$ above to hold (cf. also Example 4 at the end of this section).

(2.6) Property $\left(P_{k}\right)$. There exists a set $Z_{1}, \ldots, Z_{m}$ of non-Liouville vectors in $\mathfrak{N}_{k}$ such that every rational subspace $V$ of $\mathfrak{N}_{k}$ of codimension 1 can be supplemented to $\mathfrak{N}_{k}$ by some $Z_{j}, 1 \leq j \leq m$, and also a set of vectors $Y_{1}, \ldots, Y_{m}, Y_{1}^{\prime}, \ldots, Y_{m}^{\prime}$ in $\mathfrak{N}_{k-1}$ such that for each $1 \leq j \leq m_{e}\left[X, Y_{j}\right]=Z_{j},\left[V, Y_{j}^{\prime}\right]=Z_{j}$ and either $\left[V, Y_{j}\right]=0$ or $\left[X, Y_{j}^{\prime}\right]=0$, for all $j=1, \ldots, m$. (By Remark 1 of $\S 0$, Property $\left(P_{k}\right)$ is invariant under rational automorphisms of $\mathfrak{N}$.) There is also, in this context, a stronger version of the rational supplementation property for the pair of vectors $X$ and $V$ (which implies $\left(P_{k}\right)$ ):

$$
\begin{array}{lll}
{\left[X, \mathfrak{N}_{k-1}\right]=\mathfrak{N}_{k} \quad \text { and }} & {\left[V, \operatorname{ker}\left(\operatorname{ad}_{X} \mid \mathfrak{N}_{k-1}\right)\right]=\mathfrak{N}_{k} \quad \text { or }} \\
{\left[V, \mathfrak{N}_{k-1}\right]=\mathfrak{N}_{k} \quad \text { and } \quad\left[X, \operatorname{ker}\left(\operatorname{ad}_{V} \mid \mathfrak{N}_{k-1}\right)\right]=\mathfrak{N}_{k} .}
\end{array}
$$

Thus we have the following complex version of Theorem 1.

THEOREM $1_{\mathbf{C}}$. If $X$ and $V \in \mathfrak{N}$, an n-step nilpotent Lie algebra, commute and have Property $\left(P_{k}\right)$ or $\left(P_{k}^{\prime}\right), k=1, \ldots, n-1$, then $D=X+i V$ is globally regular on every compact nilmanifold $\Gamma \backslash N, N=\exp \mathfrak{N}$.

We notice that the pair $X_{1}, V_{1}$ of Example 1 satisfies $\left(P_{n-1}^{\prime}\right)$. Also, the pair $X_{1}, X_{2}$ of Examples 2 and 3 satisfies $\left(P_{2}^{\prime}\right)$. In Example $2\left(P_{1}\right)$ holds but $\left(P_{1}^{\prime}\right)$ does not. In Example 3 neither $\left(P_{1}\right)$ nor $\left(P_{1}^{\prime}\right)$ is satisfied, nevertheless $D=X_{1}+i X_{2}$ there is globally regular if $X_{1}$ and $X_{2}$ are algebraic. This is because $\bar{X}_{1}$ and $\bar{X}_{2}$ both are algebraic vectors in the step 2 Lie algebra $\overline{\mathfrak{N}}=\mathfrak{N} / \mathbf{R} Z$ and we are in the situation of Theorem $2_{\mathbf{C}}$. In fact, we can state the following

COROLLARY. Let $N$ be a 3-step nilpotent Lie group possessing a cocompact discrete subgroup $\Gamma$. If $X_{1}, X_{2} \in \mathfrak{N}$ satisfy $\left(P_{2}\right)\left(\right.$ or $\left.\left(P_{2}^{\prime}\right)\right),\left[X_{1}, X_{2}\right]=0$, and both $X_{1}$ and $X_{2}$ are non-Liouville vectors (with respect to $\log \Gamma$ ), then $D=X_{1}+i X_{2}$ is globally regular on $\Gamma \backslash N$.

We end this section with the example showing that the assumption that both $X$ and $V$ have the supplementation property is not sufficient for global regularity of $D=X+i V$. 
EXAMPLE 4. Let $\mathfrak{N}$ be the 2-step nilpotent Lie algebra spanned by $X_{1}, X_{2}$, $Y, Z$ with the commutation relations generated by $\left[X_{j}, Y\right]=Z, j=1,2$. Let $N=\exp \mathfrak{N}$ and let $\Gamma$, a discrete cocompact subgroup, be such that $\log \Gamma$ contains $X_{1}, X_{2}, Y, Z$. Let $D=X_{1}+i\left(X_{2}+\beta Z\right)$, where $\beta$ is a Liouville number. Then, clearly, both $X_{1}$ and $X_{2}+\beta Z$, have supplementation. However, the algebra $\mathfrak{N}$ is in fact a direct product of a Heisenberg algebra spanned by $X_{1}, Y, Z$, and of $\mathbf{R}^{1}$ spanned by $X_{2}-X_{1}$. Therefore the center of $\mathfrak{N}$ is spanned by $Z$ and $Z_{2}=X_{2}-X_{1}$, and the infinite-dimensional representations of $N$ corresponding to $\Lambda \in \mathfrak{N}^{*}$ integral on the center act on $L^{2}(\mathbf{R})$ with $\pi_{\Lambda}\left(X_{1}\right)=\partial / \partial x, \pi_{\Lambda}(Y)=i \Lambda(Z) x, \pi_{\Lambda}(Z)=i \Lambda(Z)$, $\pi_{\Lambda}\left(Z_{2}\right)=i \Lambda\left(Z_{2}\right)$. Consequently,

$$
\pi_{\Lambda}(D)=(1+i) \frac{\partial}{\partial x}-\left[\Lambda\left(Z_{2}\right)+\beta \Lambda(Z)\right],
$$

which, after applying the Fourier transform, becomes

$$
\hat{\pi}_{\Lambda}(D)=(i-1) \xi-\left[\Lambda\left(Z_{2}\right)+\beta \Lambda(Z)\right]
$$

As in Example 1 of $\S 1$ we can show that $D$ is not globally regular on $\Gamma \backslash N$.

3. 3-step nilmanifolds, $[X, Y]=0$. In this section we will prove the following "complex combination" of Theorems 1 and 2. As we needed Property $\left(P_{k}\right)$ in Theorem $1_{\mathbf{C}}$ and $\mathfrak{N}$ to be metabelian in Theorem $2_{\mathbf{C}}$, here we also need some additional commutation properties of $\mathfrak{N}$.

THEOREM 3. Let $N$ be a 3-step nilpotent Lie group with Lie algebra $\mathfrak{N}$ and a cocompact discrete subgroup $\Gamma$. If $X, Y \in \mathfrak{N}$ commute, $X$ has the non-Liouville supplementation property, $Y \in[\mathfrak{N}, \mathfrak{N}]$ is a non-Liouville vector and moreover, $\left[\operatorname{ker}\left(\operatorname{ad}_{X}^{2}\right), Y\right]=[\mathfrak{N}, Y]$ then $D=X+i Y$ is globally regular on the compact nilmanifold $\Gamma \backslash N$.

Since the proof of Theorem 3 for some $\Lambda \in(\Gamma \backslash N)^{\wedge}$ requires the estimates on step 2 quotient groups of $N$, we will state and prove its stronger step 2 version first.

THEOREM $3^{\prime}$. Let $N$ be a step 2 nilpotent Lie group with Lie algebra $\mathfrak{N}$ and a cocompact discrete subgroup $\Gamma$. If $X, Y \in \mathfrak{N}$ commute, $X$ has the non-Liouville supplementation property, and $Y$ is a non-Liouville vector, then $D=X+i Y$ is globally regular on $\Gamma \backslash N$.

ProOF OF THEOREM $3^{\prime}$. Let $\Lambda \in(\Gamma \backslash N)$ and let $Z_{1}, \ldots, Z_{n}$ be a rational basis of $\mathfrak{Z}$. We reduce $\mathfrak{N}$ to an algebra $\overline{\mathfrak{N}}$ with 1 -dimensional center $\overline{\mathfrak{Z}}$ spanned by $\bar{Z}_{j}$, say. We may assume $\bar{Y} \notin \overline{\mathfrak{Z}}$, otherwise we would have applied the estimates from the proof of Theorem $1^{\prime}$ which are unaffected by the passage from $\mathfrak{N}$ to $\overline{\mathfrak{N}}$. (It is in using Theorem $1^{\prime}$ that we need $Y$ non-Liouville.) There exists $\bar{X}_{j} \in \overline{\mathfrak{N}}$ such that $\left[\bar{X}_{j}, \bar{Y}\right]=\bar{Z}_{j}$. We choose $X_{j}$ to be the first external vector in our construction of the Schrödinger model for $\pi_{\Lambda}$ with a special maximal subordinate to $\Lambda$ subalgebra $\mathfrak{M}$. Clearly $\bar{X} \in \mathfrak{Z}(\bar{Y})$. Two cases are now possible.

Case I. $\overline{\bar{X}} \in \mathfrak{Z}\left(\mathfrak{Z}(\bar{Y})^{-}\right)$. Then $\pi_{\Lambda}\left(X_{j}\right)=\partial / \partial x, \pi_{\Lambda}(Y)=i \Lambda\left(Z_{j}\right) x, \pi_{\Lambda}(X)=$ $i \Lambda\left(\left[X, X_{j}\right]\right) x$, and $\pi_{\Lambda}(D)=\left\{i \Lambda\left(\left[X, X_{j}\right]\right)-\Lambda\left(Z_{j}\right)\right\} x, \Lambda\left(Z_{j}\right) \neq 0$. We use the same estimates as we used in (2.1). 
Case II. $\overline{\bar{X}} \notin \mathfrak{Z}\left(\mathfrak{Z}(\bar{Y})^{-}\right)$. We choose $X$ to be the second external vector. There exists $\overline{\bar{Y}}_{j} \in \mathfrak{Z}(\bar{Y})^{-}$such that $\left[\bar{X}, \overline{\bar{Y}}_{j}\right]=\overline{\bar{Z}}_{j}$. In this model

$$
\begin{gathered}
\pi_{\Lambda}\left(X_{j}\right)=\frac{\partial}{\partial v}, \quad \pi_{\Lambda}(Y)=i \Lambda\left(Z_{j}\right) v, \quad \pi_{\Lambda}\left(Y_{j}\right)=i \Lambda\left(\left[X_{j}, Y_{j}\right]\right) v+i \Lambda\left(Z_{j}\right) x \\
\pi_{\Lambda}(X)=\frac{\partial}{\partial x}+i \Lambda\left(\left[X, X_{j}\right]\right) v, \quad \text { and } \quad \pi_{\Lambda}(D)=\frac{\partial}{\partial x}+i \Lambda\left(\left[X, X_{j}\right]\right) v-\Lambda\left(Z_{j}\right) v .
\end{gathered}
$$

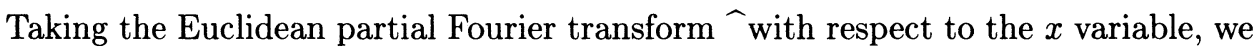
get

$$
\begin{aligned}
& \hat{\pi}_{\Lambda}\left(X_{j}\right)=\frac{\partial}{\partial v}, \quad \hat{\pi}_{\Lambda}(Y)=i \Lambda\left(Z_{j}\right) v, \quad \hat{\pi}_{\Lambda}(X)=i \xi+i \Lambda\left(\left[X, X_{j}\right]\right) v \\
& \hat{\pi}_{\Lambda}\left(Y_{j}\right)=i \Lambda\left(\left[X_{j}, Y_{j}\right]\right) v-\Lambda\left(Z_{j}\right) \frac{\partial}{\partial \xi}, \quad \text { and } \\
& \hat{\pi}_{\Lambda}(D)=i\left(\xi+\Lambda\left(\left[X, X_{j}\right]\right) v\right)-\Lambda\left(Z_{j}\right) v \equiv P_{\Lambda}(\xi, v) .
\end{aligned}
$$

As in (2.3), $\left|P_{\Lambda}(\xi, v)\right|^{2} \geq c_{\Lambda j}\left(\xi^{2}+v^{2}\right)$ for all real $\xi$ and $v$, where the lowest eigenvalue $c_{\Lambda j}$ of the quadratic form $\left|P_{\Lambda}(\xi, v)\right|^{2}$ satisfies the inequality

$$
c_{\Lambda j} \geq \Lambda\left(Z_{j}\right)^{2}\left\{\Lambda\left(\left[X, X_{j}\right]\right)^{2}+\Lambda\left(Z_{j}\right)^{2}+1\right\}^{-1} .
$$

Now we apply the argument of $\S 2$ stated after (2.3) with $\partial / \partial v=\pi_{\Lambda}\left(X_{j}\right)$ and $\partial / \partial \xi=\hat{\pi}_{\Lambda}\left\{-\Lambda\left(Z_{j}\right)^{-1}\left(Y_{j}+i \Lambda\left(Z_{j}\right)^{-1}\left[X_{j}, Y_{j}\right] Y\right)\right\}$.

PROOF OF THEOREM 3. Let $Z_{1}, \ldots, Z_{m}$ be the set of non-Liouville vectors in $\mathfrak{N}_{2}$ described in Definition (0.2) and let $\Lambda \in(\Gamma \backslash N)$ be such that $\Lambda\left(Z_{j}\right) \neq 0$ for some $1 \leq j \leq m$. (Otherwise we would have applied Theorem $3^{\prime}$.) We may assume that $\bar{Y}$ is not in the center of $\overline{\mathfrak{N}}$. (Otherwise we would use the estimates from the proof of Theorem $1^{\prime}$, which are unaffected by the passage from $\mathfrak{N}$ to $\overline{\mathfrak{N}}$.) Hence there exists $\bar{X}_{j}$ in $\overline{\mathfrak{N}} \sim[\overline{\mathfrak{N}}, \overline{\mathfrak{N}}]$ that brackets $\bar{Y}$ onto $\bar{Z}_{j}$. We choose $X_{j}$ (any preimage of $\bar{X}_{j}$ in $\left.\mathfrak{N}\right)$ in $\operatorname{ker}\left(\operatorname{ad}_{X}^{2}\right)$ to be the first external vector in our construction of a special $\mathfrak{M}_{\Lambda}$. Now $\bar{X} \in \mathfrak{Z}(\bar{Y})$. By the non-Liouville supplementation property of $X$, there exists $Y_{j} \in[\mathfrak{N}, \mathfrak{N}]$ such that $\left[X, Y_{j}\right]=Z_{j}$. We notice that $\overline{\bar{Y}}_{j} \in \mathfrak{Z}(\bar{Y})^{-}$since $Y$ and $Y_{j}$, both being in $[\mathfrak{N}, \mathfrak{N}]$ of a step 3 algebra $\mathfrak{N}$, commute. Also, $\left[\overline{\bar{Y}}_{j}, \mathfrak{Z}(\bar{Y})^{-}\right]=\mathbf{R} \overline{\bar{Z}}_{j}$, the center of $\mathfrak{Z}(\bar{Y})^{-}$. Thus $X$ can be chosen to be the second external vector. Let $N=\exp \mathfrak{M}_{\Lambda} \cdots \exp \mathbf{R} S_{k} \cdots \exp \mathbf{R} X \exp \mathbf{R} X_{j}$ be the parametrization of $N$ obtained by this construction. $\Lambda$ has already been used in passing to the quotient algebra $\overline{\mathfrak{N}}$. However, by applying $\operatorname{Ad}^{*} \exp t \bar{X}_{j}$, we can make $\Lambda(Y)=0$, and since $\bar{X} \in \mathfrak{Z}(\bar{Y})$, we will not disturb this arrangement if we use $\operatorname{Ad}^{*} \exp t^{\prime} \bar{X}$ to make $\Lambda\left(Y_{2}\right)=0$ too. Also, $\Lambda$ remains rational on the center. Thus we have

$$
\pi_{\Lambda}\left(X_{j}\right)=\frac{\partial}{\partial v}, \quad \pi_{\Lambda}(Y)=i \Lambda\left(Z_{j}\right) v, \quad \pi_{\Lambda}\left(Y_{j}\right)=i \Lambda\left(\left[X_{j}, Y_{j}\right]\right) v+i \Lambda\left(Z_{j}\right) x
$$

and

$$
\pi_{\Lambda}(X)=\frac{\partial}{\partial x}+i\left\{\frac{1}{2} v^{2} \Lambda\left(\left[\left[X_{j}, X\right], X_{j}\right]\right)+v\left(\sum_{k=1}^{l} s_{k} \Lambda\left(\left[\left[X, X_{j}\right] S_{k}\right]\right)+\Lambda\left(\left[X, X_{j}\right]\right)\right)\right\}
$$

(Here we use the fact that $\mathfrak{M}_{\Lambda}$ can be taken both to be special and to contain $[\mathfrak{N}, \mathfrak{N}]$ simultaneously.) Taking the Euclidean partial Fourier transform with respect to the 
$x$-variable we obtain

$$
\hat{\pi}_{\Lambda}\left(X_{j}\right)=\frac{\partial}{\partial v}, \quad \hat{\pi}_{\Lambda}(Y)=i \Lambda\left(Z_{j}\right) v, \quad \hat{\pi}_{\Lambda}\left(Y_{j}\right)=i \Lambda\left(\left[X_{j}, Y_{j}\right]\right) v-\Lambda\left(Z_{j}\right) \frac{\partial}{\partial \xi}
$$

and

$$
\hat{\pi}_{\Lambda}(X)=i\left\{\xi+\frac{1}{2} v^{2} \Lambda\left(\left[\left[X_{j}, X\right] X_{j}\right]\right)+v\left(\sum_{k=1}^{l} s_{k} \Lambda\left(\left[\left[X, X_{j}\right], S_{k}\right]\right)+\Lambda\left(\left[X, X_{j}\right]\right)\right)\right\} .
$$

Hence $\partial / \partial v=\hat{\pi}_{\Lambda}\left(X_{j}\right), \partial / \partial \xi=\hat{\pi}_{\Lambda}\left\{-\Lambda\left(Z_{j}\right)^{-1}\left(i \Lambda\left(Z_{j}\right)^{-1}\left[X_{j}, Y_{j}\right] Y+Y_{j}\right)\right\}$ and $\hat{\pi}_{\Lambda}(D)$ acts on $L^{2}\left(\mathbf{R}^{l+2}\right)$ as a multiplication by a polynomial $P_{\Lambda j}\left(s_{\Lambda}, \xi, v\right)$.

Letting

$$
A=A(v)=\frac{1}{2} v \Lambda\left(\left[\left[X_{j}, X\right], X_{j}\right]\right)+\sum_{k=1}^{l} s_{k} \Lambda\left(\left[\left[X, X_{j}\right], S_{k}\right]\right)+\Lambda\left(\left[X, X_{j}\right]\right),
$$

we get

$$
\left|P_{\Lambda j}\left(s_{\Lambda}, \xi, v\right)\right|^{2}=\Lambda\left(Z_{j}\right)^{2} v^{2}+(A v+\xi)^{2}=Q(A(v), v, \xi)
$$

Introducing a dummy variable $v^{1}$, and letting $c_{\Lambda, j}\left(v^{1}\right)$ be the minimum eigenvalue of the quadratic form $Q\left(A\left(v^{1}\right), v, \xi\right)$ in $(v, \xi)$, we have $Q\left(A\left(v^{1}\right), v, \xi\right) \geq$ $c_{\Lambda, j}\left(v^{1}\right)\left(v^{2}+\xi^{2}\right)$. Now we let $v^{1}=v$, and we use (2.3) with $a=\Lambda\left(Z_{j}^{2}\right)+A^{2}, b=A$, and $c=1$ to calculate $c_{\Lambda, j}$. The radical in the resulting denominator in (2.3) is less than $\Lambda\left(Z_{j}\right)^{2}+A^{2}+1$, so we have $c_{\Lambda, j} \geq \Lambda\left(Z_{j}\right)^{2}\left(\Lambda\left(Z_{j}\right)^{2}+A^{2}+1\right)^{-1}$.

We get the $L^{2}\left(\mathbf{R}^{l+2}\right)$ estimates on $\tilde{f}_{\Lambda}$ in essentially the same manner as in (2.3) of $\S 2$, the only difference being that in (2.4), before taking the sup, instead of $M_{j}$ of $\left(1.1^{\prime}\right)$ we use (1.1) and we write the multiplication by $A$ as an action of $\hat{\pi}_{\Lambda}\left(U_{j}\right)+\Lambda\left(Z_{j}\right)^{-1} \hat{\pi}_{\Lambda}\left(V_{j}\right)$ for some $U_{j}, V_{j} \in \mathcal{U}(\mathfrak{N})$. Since $\hat{\pi}_{\Lambda}(-i X)=A \cdot v+\xi$, and $\hat{\pi}_{\Lambda}\left(X_{j}\right)=\partial / \partial v$, we have

$$
\hat{\pi}_{\Lambda}\left(\left[X_{j},-i X\right]\right)=v \frac{\partial}{\partial v} A+A .
$$

But $v(\partial / \partial v) A=(v / 2) \Lambda\left(\left[\left[X_{1}, X\right], X_{j}\right]\right)$ so

$$
A=\hat{\pi}_{\Lambda}\left(\left[X_{j},-i X\right]+\frac{1}{2} \Lambda\left(Z_{j}\right)^{-1}\left[\left[X_{j}, X\right] X_{j}\right] Y\right)=\hat{\pi}_{\Lambda}\left(U_{j}\right)+\Lambda\left(Z_{j}\right)^{-1} \hat{\pi}_{\Lambda}\left(V_{j}\right),
$$

because $\hat{\pi}_{\Lambda}\left(\left[\left[X_{j}, X\right], X_{j}\right]\right)=i \Lambda\left(\left[\left[X_{j}, X\right], X_{j}\right]\right)$, and $\pi_{\Lambda}(Y)=i \Lambda\left(Z_{j}\right) v$.

REMARK 1. The assumption $\left[\operatorname{ker}\left(\operatorname{ad}_{X}^{2}\right), Y\right]=[\mathfrak{N}, Y]$ forces $\left[\left[X, X_{j}\right], X\right]$ to be 0 in the Schrödinger model we construct. Without this assumption we would have

$$
\begin{aligned}
\pi_{\Lambda}(X)=\frac{\partial}{\partial x}+i\left\{\frac{v^{2}}{2}\right. & \left(\left[\left[X_{j}, X\right], X_{j}\right]\right)+x v \Lambda\left(\left[\left[X, X_{j}\right], X\right]\right) \\
& \left.+v\left[\sum_{k=1}^{l} s_{k} \Lambda\left(\left[\left[X, X_{j}\right], S_{k}\right]\right)+\Lambda\left(\left[X, X_{j}\right]\right)\right]\right\} .
\end{aligned}
$$

We were unable to get satisfactory estimates when the " $x v$ "-term is present; nor were we able to provide a simpler than the described above parametrization of a 3-step $\mathfrak{N}$.

REMARK 2. If in Example 2 of $\S 5$ we consider the operator $D=X_{1}+i Y_{2}$ the hypothesis on $\operatorname{ker} \operatorname{ad}_{X}^{2}$ in Theorem 3 is satisfied. 
4. 3-step nilmanifolds, $[X, Y]$ central. In this section we will prove a version of Theorems 3 and $3^{\prime}$ of the previous section for $[X, Y] \neq 0$, central. We remark that the proof of this case is quite different from that used in the case of $[X, Y]=0$. However, the case $[X, Y]=0$ must be proved first, since it is an essential preliminary to proving the case with $[X, Y] \neq 0$. The reason for this is that, even if $[X, Y] \neq 0$, there are $\Lambda \in(\Gamma \backslash N)$ such that $\Lambda([X, Y])=0$, which forces us to fall back upon the commuting case which is already proved. Also, Part II of the proof of Theorem 4 in the present paper corrects an oversight in part (B) of the proof of Theorem (3.13) in [5]. The right-hand side of inequality (3.16) on p. 548 there should have contained terms of the form $\left|\tilde{U}_{n} \cdots \tilde{U}_{1} \tilde{f}_{\Lambda, q}\left(s_{\Lambda}, 0\right)\right|$. Consequently, what we call Lemma 1 in this section is missing there.

THEOREM 4. Let $N$ be a step 3 or step 2 nilpotent Lie group with Lie algebra $\mathfrak{N}$ and a cocompact discrete subgroup $\Gamma$. If $X, Y \in \mathfrak{N}$, if $X$ has the non-Liouville supplementation property, and $Y$ is in the next to the last step of the lower central series of $\mathfrak{N}$ (which is the commutator $[\mathfrak{N}, \mathfrak{N}]$ if $\mathfrak{N}$ is of step 3 and $\mathfrak{N}$ itself if $\mathfrak{N}$ is of step 2), then $D=X+i Y$ is globally regular on the compact nilmanifold $\Gamma \backslash N$ provided both $Y$ and $[X, Y]$ are non-Liouville vectors. By the assumption, $Z=[X, Y]$ is central. Moreover, if $\mathfrak{N}$ is of step 3 with the center more than 1 dimensional we assume that $\left[\operatorname{ker}\left(\operatorname{ad}_{X}^{2}\right), Y\right]=[\mathfrak{N}, Y]$.

ProOF. Let $\Lambda \in(\Gamma \backslash N)^{\wedge}$ and assume $\lambda:=\Lambda(Z) \neq 0$. Otherwise we would have applied the estimates of Theorem 3 , or $3^{\prime}$ to $\tilde{f}_{\Lambda}$ on the quotient group $N / W$, where $W=\exp \mathfrak{W}$ and $\mathfrak{W}$ is the smallest rational subspace of $\mathfrak{N}$ containing $Z$. Since $\operatorname{ker}(\Lambda \mid \mathfrak{Z})$ is a rational subspace containing $Z$, it follows that $\mathfrak{W} \subset \operatorname{ker}(\Lambda \mid \mathfrak{Z})$. Since $\mathfrak{W} \subset \mathfrak{Z}, \mathfrak{W}$ is also a (rational) ideal of $\mathfrak{N}$. Notice that in case of $\mathfrak{Z}$ being 1 dimensional, $N / W$ is of step 2 and we apply Theorem $3^{\prime}$, while in case of $\operatorname{dim} \mathfrak{W}<$ $\operatorname{dim} \mathfrak{Z}, N / W$ may still be of step 3 thus forcing us to apply Theorem 3 with its additional assumption $\left[\operatorname{ker}\left(\operatorname{ad}_{X}^{2}\right), Y\right]=[\mathfrak{N}, Y]$, or actually with $\left[\operatorname{ker}\left(\operatorname{ad}_{\bar{X}}^{2}\right), \bar{Y}\right]=$ $[\overline{\mathfrak{N}}, \bar{Y}]$ where $\overline{\mathfrak{N}}=\mathfrak{N} / \mathfrak{W}$. We choose $X$ to be the first external vector in our construction of $\mathfrak{M}_{\Lambda}$, and thus we have

$$
\pi_{\Lambda}(X)=\frac{\partial}{\partial x}, \quad \pi_{\Lambda}(Y)=i \lambda x, \quad \pi_{\Lambda}(Z)=i \lambda,
$$

and $\pi_{\Lambda}(D)=\partial / \partial x-\lambda x$. Hence

$$
\tilde{f}_{\Lambda}\left(s_{\Lambda}, x\right)=\exp \left(\frac{\lambda x^{2}}{2}\right)\left(\int_{0}^{x} \tilde{g}_{\Lambda}\left(s_{\Lambda}, t\right) \exp \left(\frac{-\lambda t^{2}}{2}\right) d t+c\left(s_{\Lambda}\right)\right)
$$

where $c\left(s_{\Lambda}\right)$ is a constant depending on $s_{\Lambda}$, is the general solution of $\pi_{\Lambda}(D) \tilde{f}_{\Lambda}=\tilde{g}_{\Lambda}$. We will break the proof now into two parts, I and II, depending on whether $\lambda$ is positive or negative.

PART I $(\lambda>0)$. We are interested in the solutions $\tilde{f}_{\Lambda}$ of the form (4.1) which are in the Schwartz space $S\left(\mathbf{R}^{d}\right)$. This, in particular, means that $\lim _{x \rightarrow \pm \infty} \tilde{f}_{\Lambda}\left(s_{\Lambda}, x\right)=$ 0 , which implies

$$
c\left(s_{\Lambda}\right)=-\int_{0}^{\infty} \tilde{g}_{\Lambda}\left(s_{\Lambda}, t\right) \exp \left(\frac{-\lambda t^{2}}{2}\right) d t=-\int_{0}^{-\infty} \cdots .
$$


That is,

$$
\begin{aligned}
\tilde{f}_{\Lambda}\left(s_{\Lambda}, x\right) & =-\int_{x}^{\infty} \tilde{g}_{\Lambda}\left(s_{\Lambda}, t\right) \exp \left[\frac{\lambda\left(x^{2}-t^{2}\right)}{2}\right] d t \\
& =\int_{-\infty}^{x} \ldots
\end{aligned}
$$

As a rule, we will work with the first integral if $x \geq 0$ and with the second one if $x<0$. To get the estimates on the $L^{2}\left(\mathbf{R}^{d}\right)$ norm of $\tilde{f}_{\Lambda}$, we will need the following estimate on $\tilde{g}_{\Lambda}\left(s_{\Lambda}, x\right)$, which we obtain as in $[\mathbf{7}$, p. 545$]$ except that instead of $\delta$ there, which came from $\left(1.1^{\prime}\right)$, we use (1.1). In the notation of (1.1), but replacing $n$ by $m$ and $d$ by $k$, take the $\sup _{t}$ and obtain, by Sobolev's inequality, with Sobolev constant 2 ,

$$
\begin{aligned}
\left|\tilde{g}_{\Lambda}\left(s_{\Lambda}, t\right)\right|^{2} \leq & 2 c^{4 k} \sum_{l=0}^{1}\left\|\left(\tilde{e}_{1}^{2}+\cdots+\tilde{e}_{m}^{2}\right)^{4 k} \tilde{X}^{l}\left(\tilde{Z}^{2}+\tilde{Y}^{2}\right)^{k} \tilde{g}_{\Lambda}\left(s_{\Lambda}, \cdot\right)\right\|^{2} \\
& \cdot \begin{cases}t^{-4 k}, & \text { for }|t| \geq 1, \\
1, & \text { for }|t|<1,\end{cases}
\end{aligned}
$$

where $\left\|h\left(s_{\Lambda}, \cdot\right)\right\|^{2}=\int_{-\infty}^{\infty}\left|h\left(s_{\Lambda}, t\right)\right|^{2} d t$. Of course (4.3) has nothing to do with solvability of $\hat{\pi}(D) \hat{f}_{\Lambda}=\hat{g}_{\Lambda}$, and works for any $\hat{g}_{\Lambda} \in S\left(\mathbf{R}^{d}\right)$; thus it is also true for $\tilde{U} \tilde{g}_{\Lambda}$, for all $U \in \mathcal{U}(\mathfrak{N})$.

Now let $\mathfrak{N} \supset \mathfrak{N}_{1} \supset \cdots \supset \mathfrak{N}_{k} \supset\{0\}$ be the lower central series of $\mathfrak{N}$ : so $\left[\mathfrak{N}, \mathfrak{N}_{i}\right] \equiv$ $\mathfrak{N}_{i+1}$. If $U \in \mathfrak{N}$, define the rank of $U$ to be $k-i$, where $i=\operatorname{Max}\left\{j \mid U \in \mathfrak{N}_{j}\right\}$. Clearly $\operatorname{rank}([U, V])<\operatorname{rank} U$ for any $U, V \in \mathfrak{N}$. Thus $U \in \mathfrak{Z}$ if $\operatorname{rank} U=0$. If the monomial $A=U_{m} \cdots U_{1} \in \mathcal{U}(\mathfrak{N})=$ the universal enveloping algebra of $\mathfrak{N}$, define the rank of (the factorization of) $A$ to be $\sum_{1}^{m} \operatorname{rank} U_{i}$.

If $D f_{\Lambda, q}=g_{\Lambda, q}$, then

$$
D\left(U_{n} \cdots U_{1} f_{\Lambda, q}\right)=U_{n} \cdots U_{1} g_{\Lambda, q}+\sum_{l=1}^{n} U_{n} \cdots U_{l+1}\left[D, U_{l}\right] U_{l-1} \cdots U_{1} f_{\Lambda, q}
$$

and

$$
\operatorname{rank}\left(U_{n} \cdots U_{l+1}\left[D, U_{l}\right] U_{l-1} \cdots U_{1}\right)<\operatorname{rank}\left(U_{n} \cdots U_{1}\right) .
$$

Observe that rank $\left(U_{n} \cdots U_{1}\right)=0 \Rightarrow U_{n} \cdots U_{1} \in \mathcal{U}(\mathfrak{Z})$.

Inductive hypothesis. If $\operatorname{rank}\left(U_{n} \cdots U_{1}\right) \leq N$, then, for each $k=1,2,3, \ldots$,

$$
\begin{aligned}
& \left|\tilde{U}_{n} \cdots \tilde{U}_{1} \tilde{f}_{\Lambda, q}\left(s_{\Lambda}, x\right)\right| \leq \sum_{l=1}^{N+1} \operatorname{Min}\left(1, x^{-4 k+l}\right) \\
& \quad \cdot \sum_{p} c_{p l}\left(\sum_{r=0}^{1}\left\|\tilde{X}^{r}\left(\tilde{e}_{1}^{2}+\cdots+\tilde{e}_{m}^{2}\right)^{4 k}\left(\tilde{Z}^{2}+\tilde{Y}^{2}\right)^{k} \tilde{U}_{p l} \tilde{g}_{\Lambda}\left(s_{\Lambda}, \cdot\right)\right\|^{2}\right)^{1 / 2}
\end{aligned}
$$

where the sum $\sum_{p}$ is finite, and $\operatorname{rank} U_{p l}=l$. (In fact, each $U_{p l}$ is a product of $U_{j}$ 's, $\left[D, U_{j}\right]$ 's and $\left[D\left[D U_{j}\right]\right]$ 's, $1 \leq j \leq n$.) The constants $c_{p l}$ depend on $c$ of (1.1), on the Sobolev constant, on $k$, and on $U_{1}, \ldots, U_{n}$, but do not depend on $\Lambda$.

REMARK. $\left(\mathrm{I}_{N}\right)$ implies the desired estimate on the $L^{2}\left(\mathbf{R}^{d}\right)$ norm of $\tilde{U}_{n} \cdots \tilde{U}_{1} \tilde{f}_{\Lambda}$ $\left(=L^{2}(\Gamma \backslash N)\right.$ norm of $\left.U_{n} \cdots U_{1} f_{\Lambda}\right)$ if $k$ is sufficiently large-we just square $\left(\mathrm{I}_{N}\right)$ and integrate it $d s_{\Lambda} d x$ over $\mathbf{R}^{d}$. We will show now that the estimates $\left(\mathrm{I}_{N}\right)$ hold. 
Initial case. For $N=0\left(\mathrm{I}_{N}\right)$ reads

$$
\begin{aligned}
\left|\tilde{U}_{n} \cdots \tilde{U}_{1} \tilde{f}_{\Lambda}\left(s_{\Lambda}, x\right)\right| & \leq \operatorname{Min}\left(1,|x|^{-4 k+1}\right) \\
& \cdot \sum_{p} c_{p}\left(\sum_{r=0}^{1}\left\|\tilde{X}^{r}\left(\tilde{e}_{1}^{2}+\cdots+\tilde{e}_{m}^{2}\right)^{4 k}\left(\tilde{Z}^{2}+\tilde{Y}^{2}\right)^{k} \tilde{U}_{p} \tilde{g}_{\Lambda}\left(s_{\Lambda}, \cdot\right)\right\|^{2}\right)^{1 / 2},
\end{aligned}
$$

which is a square root of (4.3) combined with (4.2), plus the observation that $\exp \left[\lambda\left(x^{2}-t^{2}\right) / 2\right] \leq 1$ under the integral sign of (4.2) and that $U_{n} \cdots U_{1}$ commutes with $D$.

Inductive step. To see that $\left(\mathrm{I}_{n}\right) \Rightarrow\left(\mathrm{I}_{N+1}\right)$, let $U=U_{n} \cdots U_{1}$ be of $\operatorname{rank} N+1$. By (4.3), $\tilde{f}_{1}=\tilde{U} \tilde{f}_{\Lambda}$ is the unique Schwartz solution of

$$
\pi_{\Lambda}(D) \tilde{f}_{1}=\tilde{U} \tilde{g}_{\Lambda}+\sum_{q=1}^{n} \tilde{U}_{n} \cdots\left[\tilde{D}, \tilde{U}_{q}\right] \cdots \tilde{U}_{1} \tilde{f}_{\Lambda} \equiv \tilde{h}_{\Lambda}
$$

and therefore is given by $(4.2)$ with $\tilde{g}_{\Lambda}$ there replaced by the above $\tilde{h}_{\Lambda}$. That is,

$$
\begin{array}{r}
\tilde{U} \tilde{f}_{\Lambda}\left(s_{\Lambda}, x\right)=\int_{-\infty}^{x}\left\{\tilde{U} \tilde{g}_{\Lambda}\left(s_{\Lambda}, t\right)+\sum_{q=1}^{n} \tilde{U}_{n} \cdots\left[\tilde{D}, \tilde{U}_{q}\right] \cdots \tilde{U}_{1} \tilde{f}_{\Lambda}\left(s_{\Lambda}, t\right)\right\} \\
\cdot \exp \left[\lambda\left(x^{2}-t^{2}\right) / 2\right] d t .
\end{array}
$$

The first term of the above sum is estimated by an expression of the same form as the one on the right-hand side of the inequality $\left(\mathrm{I}_{0}\right)$. For the other terms we apply $\left(\mathrm{I}_{N}\right)$ to each $\tilde{U}_{n} \cdots\left[\tilde{D}, \tilde{U}_{q}\right] \cdots \tilde{U}_{1} \tilde{f}_{\Lambda}, q=1, \ldots, n$ (which are of rank $\leq N$ ), and we group the terms. The integration with respect to $t$ of $\operatorname{Min}\left(1,|t|^{-2 k+l}\right)$ in (4.4) changes the constants $c_{l p}$ and increases $l$ by 1 .

PART II $(\lambda<0)$. Here we can pick $c\left(s_{\Lambda}\right)$ of $(4.1)$ to be 0 , so that

$$
\tilde{f}_{\Lambda}\left(s_{\Lambda}, x\right)=\int_{0}^{x} \tilde{g}_{\Lambda}\left(s_{\Lambda}, t\right) \exp \left[\frac{\lambda\left(x^{2}-t^{2}\right)}{2}\right] d t .
$$

Since we have picked a solution, we do not know a priori that the function $\tilde{f}_{\Lambda}$ defined by (4.6) is Schwartz. Clearly it is in $C^{\infty}\left(\mathbf{R}^{d}\right)$. We know, by the hypothesis (cf. Definition (0.1)), that there is a function $c\left(s_{\Lambda}\right)$ for which the function $\tilde{f}_{\Lambda}^{c}$ corresponding to $c\left(s_{\Lambda}\right)$ under (4.1) is Schwartz. And $\tilde{f}_{\Lambda}$ defined by (4.6) differs from $\tilde{f}_{\Lambda}^{c}$ by $\exp \left(\lambda x^{2} / 2\right) c\left(s_{\Lambda}\right)$. Clearly, $c\left(s_{\Lambda}\right)=\tilde{f}_{\Lambda}^{c}\left(s_{\Lambda}, 0\right)$ is Schwartz on $\mathbf{R}^{d-1}$. Hence $\exp \left(\lambda x^{2} / 2\right) c\left(s_{\Lambda}\right) \in S\left(\mathbf{R}^{d}\right)$, and $\tilde{f}_{\Lambda}$ must be Schwartz being a difference of two Schwartz functions. Now (4.4) and (4.1) yield the following expression for $\tilde{U} \tilde{f}_{\Lambda}$, $U=U_{n} \cdots U_{1} \in U(\mathfrak{N}), \operatorname{rank}(U)=N$ :

$$
\begin{aligned}
\tilde{U} \tilde{f}_{\Lambda}\left(s_{\Lambda}, x\right)= & \int_{0}^{x}\left\{\tilde{U} \tilde{g}_{\Lambda}\left(s_{\Lambda}, t\right)+\sum_{q=1}^{n} \tilde{U}_{n} \cdots\left[\tilde{D}, \tilde{U}_{q}\right] \cdots \tilde{U}_{1} \tilde{f}_{\Lambda}\left(s_{\Lambda}, t\right)\right\} \\
& +\tilde{U} \tilde{f}_{\Lambda}\left(s_{\Lambda}, 0\right) \exp \left(\lambda x^{2} / 2\right) .
\end{aligned}
$$

Taking the sup over $t$ of each of the summands of the integrand in $\left(4.4^{\prime}\right)$, next applying the integral estimate

$$
\left|\int_{0}^{x} \exp \left[\frac{\lambda\left(x^{2}-t^{2}\right)}{2}\right] d t\right| \leq 1-\lambda^{-1} \quad(\lambda<0, x \in \mathbf{R})
$$


and then the Sobolev inequality (1.6) to $\sup _{t}\left|\tilde{U} \tilde{g}_{\Lambda}\left(s_{\Lambda}, t\right)\right|$, we obtain

$$
\begin{aligned}
\left|\tilde{U} \tilde{f}_{\Lambda}\left(s_{\Lambda}, x\right)\right| \leq & \left(1-\lambda^{-1}\right) \sup _{t}\left(\left|\tilde{U} \tilde{g}_{\Lambda}\left(s_{\Lambda}, t\right)\right|+\sum_{q=1}^{n}\left|\tilde{U}_{n} \cdots\left[\tilde{D}, \tilde{U}_{q}\right] \cdots \tilde{U}_{1} \tilde{f}_{\Lambda}\left(s_{\Lambda}, t\right)\right|\right) \\
& +\tilde{U} \tilde{f}_{\Lambda}\left(s_{\Lambda}, 0\right) \exp \left(\frac{\lambda x^{2}}{2}\right) \\
\leq & \left(1-\lambda^{-1}\right)\left(2 \sum_{r=0}^{1}\left\|\tilde{X}^{r} \tilde{U}_{\tilde{g}_{\Lambda}}\left(s_{\Lambda}, \cdot\right)\right\|^{2}+\sum_{q=1}^{n} \sup _{t}\left|\tilde{U}_{n} \cdots\left[\tilde{D}_{,} \tilde{U}_{q}\right] \cdots \tilde{U}_{1} \tilde{f}_{\Lambda}\left(s_{\Lambda}, t\right)\right|\right) \\
& +\tilde{U} \tilde{f}_{\Lambda}\left(s_{\Lambda}, 0\right) \exp \left(\lambda x^{2} / 2\right) .
\end{aligned}
$$

Next we return to $\left(4.5^{\prime}\right)$ for each summand on the right involving $\tilde{f}_{\Lambda}\left(s_{\Lambda}, t\right)$. We iterate this procedure until there is no $\tilde{f}_{\Lambda}\left(s_{\Lambda}, t\right)$ on the right-hand side. Then we obtain

$$
\begin{aligned}
\left|\tilde{U} \tilde{f}_{\Lambda}\left(s_{\Lambda}, x\right)\right| \leq & \sum_{l=0}^{N}\left(1-\lambda^{-1}\right)^{N-l} \sum_{p} c_{p l} \\
& \cdot\left\{\left(1-\lambda^{-1}\right)\left(\sum_{r=0}^{1}\left\|\tilde{X}^{r} \tilde{U}_{p l} \tilde{g}_{\Lambda}\left(s_{\Lambda}, \cdot\right)\right\|^{2}\right)^{1 / 2}+\left|\tilde{U}_{p l} \tilde{f}_{\Lambda}\left(s_{\Lambda}, 0\right)\right|\right\}
\end{aligned}
$$

where the sum $\sum_{p}$ is finite, $\operatorname{rank} U_{p l}=l$ (each $U_{p l}$ being a product of $U_{j}$ 's, $\left[D, U_{j}\right]$ 's and $\left.\left[D\left[D, U_{j}\right]\right], 1 \leq j \leq n\right)$, and the constants $c_{p l}$ do not depend on $\Lambda$.

To get an $L^{2}$-estimate on $\tilde{U} \tilde{f}_{\Lambda}$, we add two copies of $(4.7)$, one with $\tilde{Y}^{2} \tilde{U}$ in place of $\tilde{U}$, and the other with $\tilde{Z}^{2} \tilde{U}$ replacing $\tilde{U}$. This gives the $\lambda^{2}\left(1+x^{2}\right)$ factor on the left-hand side of the following inequality (4.8):

$$
\left|\left(\lambda^{2}+\lambda^{2} x^{2}\right) \tilde{U} \tilde{f}_{\Lambda}\left(x, s_{\Lambda}\right)\right| \leq\left\|\tilde{Z}^{2} \tilde{U} \tilde{f}_{\Lambda}\left(\cdot, s_{\Lambda}\right)\right\|_{\infty}+\left\|\tilde{Y}^{2} \tilde{U} \tilde{f}_{\Lambda}\left(\cdot, s_{\Lambda}\right)\right\|_{\infty}
$$

$\leq$ a finite linear combination (with coefficients of the form const. $\lambda^{-l}$ ) of the terms of the form $\left\|\tilde{X}^{r} \tilde{U}_{l p} \tilde{g}_{\Lambda}\left(\cdot, s_{\Lambda}\right)\right\|, r=0,1$, and of the form $\left|\tilde{U}_{p l} \tilde{f}_{\Lambda}\left(s_{\Lambda}, 0\right)\right|$.

Next, we square $(4.8)$, divide by $\lambda^{4}\left(1+x^{2}\right)^{2}$, which allows us to integrate the right-hand side of the so transformed (4.7) $d x d s_{\Lambda}$ over $\mathbf{R}^{d}$ to get

$\left\|\tilde{U} \tilde{f}_{\Lambda}\right\|^{2} \leq$ finite linear combination of $\left\|\tilde{X}^{r} \tilde{U}_{l, p} \tilde{g}_{\Lambda}\right\|^{2} \cdot \lambda^{-2 q}$

$$
\text { and of } \int_{\mathbf{R}^{d-1}}\left|\tilde{U}_{l p} \tilde{f}_{\Lambda}\left(s_{\Lambda}, 0\right)\right|^{2} d s_{\Lambda} \cdot \lambda^{-2 q} \text {. }
$$

By (1.1), $\lambda^{-1}$ can be absorbed into $U_{p l}$. Since $\sum_{\Lambda}\left\|\tilde{X}^{r} \tilde{U}_{l q} \tilde{g}_{\Lambda}\right\|^{2}<\infty$, for $g \in$ $C^{\infty}(\Gamma \backslash N)$, all we need to show to complete the proof of Theorem 4 is that $\sum_{\Lambda}\left\|\tilde{U} \tilde{f}_{\Lambda}\left(s_{\Lambda}, 0\right)\right\|_{L^{2}\left(s_{\Lambda}\right)}^{2}<\infty$ for every fixed $U \in U(\mathfrak{N})$.

LEMMA 1. $\sum_{\Lambda} \int_{\mathbf{R}^{d-1}}\left|\tilde{U} \tilde{f}_{\Lambda}\left(s_{\Lambda}, 0\right)\right|^{2} d s_{\Lambda}<\infty$, for all $U \in \mathcal{U}(\mathfrak{N})$.

We will reduce that proof of Lemma 1 to the following

LEMMA 2. $\sum_{\Lambda} \int_{\mathbf{R}^{d-1}}\left|\tilde{U} \tilde{g}_{\Lambda}\left(s_{\lambda}, 0\right)\right|^{2} d s_{\Lambda}<\infty$, for every $U \in \mathcal{U}(\mathfrak{N})$ and $g \in$ $C^{\infty}(\Gamma \backslash N)$. 
PROOF OF LEMMA 2.

$$
\begin{aligned}
\int\left|\tilde{U} \tilde{g}_{\Lambda}\left(s_{\Lambda}, 0\right)\right|^{2} d s_{\Lambda} & \leq \int \sup _{t}\left|\tilde{U} \tilde{g}_{\Lambda}\left(s_{\Lambda}, t\right)\right|^{2} d s_{\Lambda} \\
& \leq \int c \sum_{l=0}^{1}\left\|\tilde{X}^{l} \tilde{U} \tilde{g}_{\Lambda}\left(s_{\Lambda}, \cdot\right)\right\|^{2} d s_{\Lambda}=c \sum_{l=0}^{1}\left\|\tilde{X}^{l} \tilde{U} \tilde{g}_{\Lambda}\right\|^{2} .
\end{aligned}
$$

Since $X^{l} U g \in C^{\infty}(\Gamma \backslash N)$, the sum over $\Lambda \in(\Gamma \backslash N)$ of the above inequality is finite. (We notice that $\sup _{t}\left|\tilde{U} \tilde{g}_{\Lambda}\left(t, s_{\Lambda}\right)\right|$ is $s_{\Lambda}$-measurable.)

PROOF THAT LEMMA $2 \Rightarrow$ LEMma 1 . First we observe that any $U \in \mathfrak{N}$ can be written $\bar{U}=a \bar{X}+\bar{W}$ for some $a \in \mathbf{R}$ and $\bar{W} \in \mathfrak{Z}(\bar{Y})$. Let $U=U_{m} \cdots U_{1} \in \mathcal{U}(\mathfrak{N})$ with $\bar{U}_{j}=a_{j} \bar{X}+\bar{W}_{j}, a_{j} \in \mathbf{R}, \bar{W}_{j} \in \mathfrak{Z}(\bar{Y}), j=1, \ldots, m$. Then

$$
\begin{aligned}
\bar{U}= & \left(a_{m} \bar{X}+\bar{W}_{m}\right) \cdots\left(a_{1} \bar{X}+\bar{W}_{1}\right) \\
= & \text { a (finite) linear combination of mixed products } \\
& \text { of } \bar{X} \text { 's and } \bar{W}_{j} \text { 's } \\
= & \text { a (finite) linear combination of (ordered) products } \\
& \text { of the form } \bar{V}_{p} \cdots \bar{V}_{1} \bar{X}^{k} \text { with } k \geq 0, \\
& \text { and some } \bar{V}_{j} \in \mathfrak{Z}(\bar{Y})\left(V_{j} \text { not necessarily }=W_{j}\right) \\
& \text { since } \mathfrak{Z}(Y) \text { is an ideal in } \overline{\mathfrak{N}} .
\end{aligned}
$$

Therefore it is sufficient to prove Lemma 1 for $U \in \mathcal{U}(\mathfrak{N})$ of the form $U=$ $V_{p} \cdots V_{2} V_{1} X^{k}, k \geq 0, \bar{V}_{j} \in \mathfrak{Z}(\bar{Y})$, and in fact we will show that

LEMMA 3. $\tilde{V}_{p} \cdots \tilde{V}_{1} \tilde{X}^{k} \tilde{f}_{\Lambda}\left(s_{\Lambda}, 0\right)=\tilde{\nu} \tilde{g}_{\Lambda}\left(s_{\Lambda}, 0\right)$ for some $\nu \in \mathcal{U}(\mathfrak{N}), \tilde{f}_{\Lambda}$ given by (4.6), and $\bar{V}_{j} \in \mathfrak{Z}(\bar{Y}), 1 \leq j \leq p$.

Step 1. $\tilde{X} \tilde{Y}^{p} \tilde{f}_{\Lambda}=$ a linear combination of the terms of the form $\tilde{Z}^{r} \tilde{Y}^{q} \tilde{f}_{\Lambda}, q \leq$ $p+1$, and $\tilde{Y}^{p} \tilde{g}_{\Lambda}$.

PROOF OF STEP 1 (INDUCTION ON $p$ ). For $p=0$, it follows from (4.0) and (4.6) that $\tilde{X} \tilde{f}_{\Lambda}=\tilde{g}_{\Lambda}-i \tilde{Y} \tilde{f}_{\Lambda}$. Next we notice that $X Y^{p+1}=(Y X+[X, Y]) Y^{p}=$ $Y\left(X Y^{p}\right)+Z Y^{p}$ and we apply the inductive hypothesis to $X Y^{p}$.

Step 2.

$$
\tilde{X}^{k} \tilde{f}_{\Lambda}=\tilde{U} \tilde{g}_{\Lambda}+\text { a finite linear combination of } \tilde{Y}^{p} \tilde{Z}^{r} \tilde{f}_{\Lambda},
$$

for some $U \in \mathcal{U}(\mathfrak{N})$.

Proof OF STEP 2 (IndUCTION ON $k$ ). For $k=1$ this is just the $p=0$ case of the proof of Step 1. All we need to check now is that $\tilde{X}\left(\tilde{Y}^{p} \tilde{Z}^{r} \tilde{f}_{\Lambda}\right)$ is again of the form (4.9), but this is just Step 1 applied to $\tilde{Z}^{r} \tilde{f}_{\Lambda}$.

Step 3. $\left(\tilde{V}_{p} \cdots \tilde{V}_{1} \tilde{f}_{\Lambda}\right)\left(s_{\Lambda}, 0\right)=0$.

PROOF OF STEP 3. In our Schrödinger model for $\pi_{\Lambda}$, since $\mathfrak{M}_{\Lambda}$ contains the commutator $[\mathfrak{N}, \mathfrak{N}]$, each $\tilde{V}_{j}$ is of the form $\tilde{V}_{j}=\varepsilon_{j}\left(\partial / \partial v_{j}\right)+i P_{j}\left(s_{\Lambda}, x\right)$, where $\partial / \partial v_{j}$ is a directional derivative in the $s_{\Lambda}$-space, $P_{j}$ is a real polynomial, and $\varepsilon_{j}=1$ if $V_{j} \notin \mathfrak{M}_{\Lambda}$ and $\varepsilon_{j}=0$ if $V_{j} \in \mathfrak{M}_{\Lambda}$. Hence

$$
\tilde{V}_{j} \tilde{f}_{\Lambda}\left(s_{\Lambda}, x\right)=\int_{0}^{x} \varepsilon_{j}\left(\frac{\partial \tilde{g}_{\Lambda}}{\partial v_{j}}\right)\left(s_{\Lambda}, t\right) \cdot \exp \left[\frac{\lambda\left(x^{2}-t^{2}\right)}{2}\right] d t+i P_{j}\left(s_{\Lambda}, x\right) \tilde{f}_{\Lambda}\left(s_{\Lambda}, x\right),
$$


and clearly $\tilde{V}_{p} \cdots \tilde{V}_{1} \tilde{f}_{\Lambda}\left(s_{\Lambda}, 0\right)=0$, since $\tilde{f}_{\Lambda}$ itself is given by (4.5).

PROOF OF LEMMA 3 . Since all the $\tilde{V}_{j}$ 's commute with $\tilde{Y}$, by Step 2, we have

$$
\begin{aligned}
\tilde{V}_{p} \cdots \tilde{V}_{1} \tilde{X}^{k} \tilde{f}_{\Lambda}\left(s_{\Lambda}, 0\right)= & \tilde{V}_{p} \cdots \tilde{V}_{1}\left(\tilde{U}_{g_{\Lambda}}+\text { a linear combination of } \tilde{Y}^{p} \tilde{Z}^{r} \tilde{f}_{\Lambda}\right)\left(s_{\Lambda}, 0\right) \\
= & \tilde{V}_{p} \cdots \tilde{V}_{1} \tilde{U} \tilde{g}_{\Lambda}\left(s_{\Lambda}, 0\right) \\
& + \text { a linear combination of }\left(\tilde{Y}^{p}\left(\tilde{V}_{p} \cdots \tilde{V}_{1} \tilde{Z}^{r} \tilde{f}_{\Lambda}\right)\right)\left(s_{\Lambda}, 0\right) \\
& \quad \text { with } p \geq 1 \\
& + \text { a linear combination of } \tilde{V}_{p} \cdots \tilde{V}_{1} \tilde{Z}^{r} \tilde{f}_{\Lambda}\left(s_{\Lambda}, 0\right) .
\end{aligned}
$$

In the above sum the last term is zero by Step 3, and the second term is zero since $\tilde{Y}$ is just a multiplication by $i \lambda x$.

COROLlaRY 1. Let $N$ be a simply connected 2-step nilpotent Lie group, $\Gamma$ a cocompact, discrete subgroup of $N$. If $X, Y \in \mathfrak{N}$ both are algebraic (resp. nonLiouville) vectors, then $D=X+i Y$ is globally regular on $\Gamma \backslash N$ (resp. provided $[X, Y]$ is a non-Liouville vector too $)$.

Proof. Let $\Lambda \in(\Gamma \backslash N)$. If $\Lambda([X, Y])=0$, this is Proposition 1 of $\S 2$ applied to $N / \exp \mathfrak{W}$, where $\mathfrak{W}$ is the smallest rational subspace of $\mathfrak{N}$ containing $Z$. If $\Lambda([X, Y]) \neq 0$, after reducing $\mathfrak{N}$ to $\overline{\mathfrak{N}}$ with 1 -dimensional center spanned by $\bar{Z}=$ $[X, Y]^{-}$, either $\bar{X}$ or $\bar{Y}$ can be used as the first external vector and we obtain the estimates now as in the proof of Theorem 4. (If $X$ and $Y$ are algebraic, then $[X, Y]$ is algebraic too.)

COROLlaRY 2. Let $N$ be a simply connected 2-step nilpotent Lie group, and $\Gamma$ a cocompact, discrete subgroup of $N$. If $X, Y \in \mathfrak{N}$ satisfy $\left(P_{1}\right)$, then $D=X+i Y$ is globally regular on $\Gamma \backslash N$ provided $[X, Y]$ is a non-Liouville vector.

Proof. If $\Lambda([X, Y])=0$ for $\Lambda \in(\Gamma \backslash N)$, then this is Theorem $1_{\mathbf{C}}$ applied to $N / \exp \mathfrak{W}, \mathfrak{W}$ being the smallest rational subspace of $\mathfrak{N}$ containing $[X, Y]$, as in the beginning of the proof of Theorem 4 . If $\Lambda([X, Y]) \neq 0$, we choose $X$ to be the first external vector and the rest of the proof is the same as that of Theorem 4 .

5. Examples and further developments. In [7] we gave an example, based on a suggestion by Corwin (cf. [4]), to show how global regularity could fail in a 3-step group for $D=X+i Y$, where $X$ and $Y$ generate the 3 -step chain group. If the Kirillov orbits had been flat, then that parameter, summation over which yields the counterexample, would have been eliminated. In our next example, we show however that flattening the Kirillov orbits, by the addition of 1 to the dimension of the group [20] does not correct this analytic failure. (This should be compared also with Example 2 in $§ 1$.) Thus the fact that all 2-step groups have flat orbits does not seem to be relevant to the problem of global regularity.

EXAMPLE 1. Let $\mathfrak{N}$ be the Lie algebra spanned by the vectors $V, W, X, Y$, and $Z$ with all nonzero bracket products generated by $[W, X]=Y,[W, Y]=Z$, $[V, X]=Z$. Thus $\mathfrak{N}$ is of step 3 with flat Kirillov orbits and 1 -dimensional center [20]. $W$ and $X$ generate a step 3 subalgebra of $\mathfrak{N}$. Let $N=\exp \mathfrak{N}$. There is a series of representations $\pi_{\Lambda} \in \hat{N}, \lambda \in \mathbf{R} \sim\{0\}$, realized on $L^{2}\left(\mathbf{R}^{2}\right)$ in such a way that $\pi_{\lambda}(W)=\partial / \partial w$ and $\pi_{\lambda}(X)=2 \pi i \lambda\left(v+w^{2} / 3\right)[\mathbf{8}]$. Let $\Gamma$ be a cocompact discrete 
subgroup of $N$ such that $Z \in \log \Gamma$. Then $\pi_{\lambda} \in(\Gamma \backslash N)^{\wedge}$ for $\lambda= \pm 1, \pm 2, \ldots$ Let $\phi$ and $\psi$ be $C^{\infty}(\mathbf{R})$ nonnegative functions such that

$$
\begin{aligned}
& \phi(w)= \begin{cases}1 & \text { for } 0 \leq w \leq 3, \\
0 & \text { for } w \notin[-1,4],\end{cases} \\
& \psi(v)= \begin{cases}1 & \text { for }-n-1 \leq v \leq-n, \\
0 & \text { for } v \notin[-2-n,-n+1] .\end{cases}
\end{aligned}
$$

Fix $\varepsilon>0$ and $n>0$ and for each positive integer $\lambda$ define

$$
\tilde{g}_{\lambda}(w, v)=\phi(w) \psi(v) e^{2 \pi \lambda \varepsilon v}
$$

and let $g_{\lambda}, \lambda=1,2,3, \ldots$, be the corresponding functions in $C^{\infty}(\Gamma \backslash N)$, each in a different primary summand of $L^{2}(\Gamma \backslash N)$. Then $g=\sum_{\lambda} g_{\lambda}$ is smooth on $\Gamma \backslash N$, for

$$
\begin{aligned}
& \sum_{\lambda=1}^{\infty}\left\|U g_{\lambda}\right\|_{L^{2}(\Gamma \backslash N)}=\sum_{\lambda=1}^{\infty}\left\|\tilde{U} \tilde{g}_{\Lambda}\right\|_{L^{2}\left(\mathbf{R}^{2}\right)} \\
& \leq \sum_{\lambda=1}^{\infty} \|(\text { differential operator with polynomial } \\
& \quad \text { in } w, v \text { and } \lambda \text { coefficients }) \phi(w) \psi(v) \|_{2} e^{2 \pi \lambda \varepsilon(-n+1)} \\
& \leq \sum_{\lambda=1}^{\infty} \mid(\text { polynomial in } \lambda) \mid e^{2 \pi \lambda \varepsilon(-n+1)}<\infty
\end{aligned}
$$

for every fixed $U \in \mathcal{U}(\mathfrak{N})$ and $n>1$.

Let $D=W+i X$. Then

$$
\pi_{\lambda}(D)=\frac{\partial}{\partial w}-2 \pi \lambda\left(v+\frac{w^{2}}{3}\right)
$$

and

$$
\tilde{f}_{\lambda}(w, v)=-\int_{w}^{\infty} \tilde{g}_{\lambda}(t, v) \exp 2 \pi \lambda\left[\frac{\left(w^{3}-t^{3}\right)}{9}+v(w-t)\right] d t
$$

is the only Schwartz solution of $\pi_{\lambda}(D) \tilde{f}_{\lambda}=\tilde{g}_{\lambda} \cdot \tilde{f}_{\lambda}$ is indeed Schwartz, since $\tilde{g}_{\lambda}$ is compactly supported, and $\exp \left(2 \pi \lambda w^{3} / 9\right) \rightarrow 0$ as $w \rightarrow-\infty$. On the other hand, by a change of variables $t=w+u$, if $0 \leq w \leq 1$ and $-1-n \leq v \leq-n$

$$
\begin{aligned}
\left|\tilde{f}_{\lambda}(w, v)\right| & =e^{2 \pi \lambda \varepsilon v} \psi(v) \int_{0}^{\infty} \phi(w+u) \exp \left[\frac{-2 \pi \lambda\left(u^{3}+3 u^{2} w+3 u w^{2}\right)}{9}\right] e^{-2 \pi \lambda v u} d u \\
& \geq \sup \exp \left[2 \pi \lambda\left(\varepsilon v-\frac{\left(u^{3}+3 u^{2} w+3 u w^{2}\right)}{9}-v u\right]\right. \\
& \geq \exp [2 \pi \lambda(-\varepsilon-n \varepsilon-3+n)]
\end{aligned}
$$

where the sup is taken over $0 \leq w \leq 1,1 \leq u \leq 2$. For $n=5$ and $\varepsilon=\frac{1}{6}$ we thus have $\left|\tilde{f}_{\lambda}(w, v)\right| \geq \exp 2 \pi \lambda$ and $\left\|f_{\lambda}\right\|_{L^{2}(\Gamma \backslash N)}=\left\|\tilde{f}_{\lambda}\right\|_{L^{2}\left(\mathbf{R}^{2}\right)} \geq \exp 2 \pi \lambda$, i.e. $\sum_{\lambda=1}^{\infty} f_{\lambda}$ does not converge in $L^{2}(\Gamma \backslash N)$. This completes the Example 2.

Parts (a) and (b) of the next Example 2 (which in fact is an "implant" of Example 2 of $\S 1$ on the group $N$ of Example 2 of $\S 2$ ) indicate how the assumptions of Theorems $2_{\mathbf{C}}$ and 3 , respectively, can possibly be relaxed. Part (c) of Example 2 deals with Theorem 4 . 
EXAMPLE 2. Let $N$ and $\Gamma$ be as in the Example 2 of $\S 2$ and let $\Lambda \in(\Gamma \backslash N)^{\wedge}$ be such that $\Lambda(Z) \neq 0$. Then $\pi_{\Lambda}\left(X_{j}\right)=\partial / \partial x_{j}, \pi_{\Lambda}\left(Y_{j}\right)=i \Lambda(Z) x_{j}, \pi_{\Lambda}\left(U_{j}\right)=$ $i \Lambda\left(U_{j}\right)+i \Lambda(Z) x_{j}^{2} / 2, j=1,2$, and $\pi_{\Lambda}\left(U_{12}\right)=i \Lambda\left(U_{2}\right)+i \Lambda(Z) x_{1} x_{2}[\mathbf{1 7}]$. Consider now the following operators:

(a) $D_{1}=X_{1}+i\left(U_{2}+\beta_{2} Y_{2}+\gamma_{2} Z\right)$

(b) $D_{2}=\left(U_{1}+\beta_{1} Y_{1}+\gamma_{1} Z\right)+i\left(U_{2}+\beta_{2} Y_{2}+\gamma_{2} Z\right)$,

(c) $D_{3}=X_{1}-i U_{12}$.

For $\Lambda$ as above we thus have

(a) $\pi_{\Lambda}\left(D_{1}\right)=\partial / \partial x_{1}-\Lambda(Z)\left(x_{2}-x_{2+}\right)\left(x_{2}-x_{2-}\right)$,

(b) $\pi_{\Lambda}\left(D_{2}\right)=i \Lambda(Z)\left[\left(x_{1}-x_{1+}\right)\left(x_{1}-x_{1-}\right)+i\left(x_{2}-x_{2+}\right)\left(x_{2}-x_{2-}\right)\right]$ where $x_{j \pm}=$ $-\beta_{j} \pm\left(\beta_{j}^{2}-2 \gamma_{j}-2 \Lambda\left(U_{j}\right) / \Lambda(Z)\right)^{1 / 2}, j=1,2$,

(c) $\pi_{\Lambda}\left(D_{3}\right)=\partial / \partial x_{1}+\Lambda(Z) x_{1} x_{2}+\Lambda\left(U_{12}\right)$.

The operator $D_{1}$ is such that $X_{1}$ has supplementation, and $U_{2}+\beta_{2} Y_{2}+\gamma_{2} Z \notin$ $[\mathfrak{N}, \mathfrak{N}]$ and does not have supplementation. Using estimates similar to those of Example 2 of $\S 2$ one can show that $D_{1}$ is globally regular provided neither $\beta_{2}^{2} / 2-\gamma_{2}$ nor $\beta_{2}$ is a Liouville number. (The requirement on $\beta_{2}$ is needed for global regularity of $\bar{D}_{1}$ on $N / \exp \mathbf{R} Z$, which follows now from Theorem $3^{\prime}$.)

The operator $D_{2}$ is such that neither its real nor its imaginary part have supplementation, $U_{j}+\beta_{j} Y_{j}+\gamma_{j} Z \notin[\mathfrak{N}, \mathfrak{N}], j=1,2$. Again, the estimates of Example 2 and of Proposition 1 , both of $\S 2$, show that $D_{2}$ is globally regular, provided neither of the following four numbers, $\beta_{j}^{2} / 2-\gamma_{j}, \beta_{j}, j=1,2$, is Liouville.

Finally, the operator $D_{3}$ is such that $X_{1}$ has supplementation, $U_{12} \notin[\mathfrak{N}, \mathfrak{N}]$ is an algebraic vector, and $\left[X_{1}, U_{12}\right]$ is not central in $\mathfrak{N}$. Applying to $\pi_{\Lambda}(D)$ of $(\mathrm{c})$ the Euclidean partial Fourier transform with respect to the $x_{2}$ variable we obtain

$\left(\mathrm{c}^{\prime}\right) \hat{\pi}_{\Lambda}\left(D_{3}\right)=\partial / \partial x_{1}+i \Lambda(Z) x_{1} \partial / \partial \eta+\Lambda\left(U_{12}\right)$, which (for $\Lambda\left(U_{12}\right)=0$ ) is the nonhypoelliptic and nonlocally solvable operator of Mizohata [14, Appendix] and Nirenberg and Treves $[\mathbf{1 6}]$. We were unable to settle the question of global regularity of $D_{3}$. (Clearly, $\bar{D}_{3}$ is globally regular on $N / \exp \mathbf{R} Z$ by Theorem 4.)

\section{BIBLIOGRAPHY}

1. S. Agmon, Lectures on elliptic boundary value problems, Van Nostrand, Princeton, N.J., 1965.

2. L. Auslander and J. Brezin, Uniform distribution in solvmanifolds, Adv. in Math. 7 (1971), 111-144.

3. J. W. S. Cassels, An introduction to Diophantine approximation, Cambridge Univ. Press, 1965.

4. L. Corwin, A representation-theoretic criterion for local solvability of left invariant differential operators on nilpotent Lie groups, Trans. Amer. Math. Soc. 264 (1981), 113-120.

5. L. Corwin and F. P. Greenleaf, Solvability of certain left invariant differential operators by nilmanifold theory, Comm. Pure Appl. Math. 36 (1983), 755-765.

6. L. Corwin, F. Greenleaf, and R. Penney, A canonical formula for the distribution kernels of primary projections in $L^{2}$ of a nilmanifold, Comm. Pure Appl. Math. 30 (1977), 355372 .

7. J. Cygan and L. Richardson, Global solvability on two-step compact nilmanifolds, Trans. Amer. Math. Soc. 279 (1983), 537-554.

8. J. Dadok and M. Taylor, Local solvability of consatnt coefficient partial differential equa tions as a small divisor problem, Proc. Amer. Math. Soc. 82 (1981), 58-60.

9. C. S. Herz, Functions which are divergences, Amer. J. Math. 92 (1970), 641-656.

10. L. Hörmander, On the division of distributions by polynomials, Ark. Mat. 3 (1958), 555568 . 
11. N. Jacobson, Lie algebras, Interscience, New York, 1962.

12. A. Kirillov, Unitary representations of nilpotent Lie groups, Uspehi Mat. Nauk 17 (1962), 57-110; English transl., Russian Math. Surveys 17 (1962), 35-104.

13. A. Malcev, On a class of homogeneous spaces, Izv. Akad. Nauk SSSR Ser. Mat. 13 (1949), 9-32; English transl., Amer. Math. Soc. Transl. 38 (1949), 276-307.

14. S. Mizohata, Solutions nulles et solutions non analytiques, J. Math. Kyoto Univ. 1 (1962), 271-302.

15. C. Moore, Decomposition of unitary representations defined by discrete subgroups of nilpotent groups, Ann. of Math. (2) 82 (1965), 146-182.

16. L. Nirenberg and F. Treves, Solvability of a first order linear partial differential equation, Comm. Pure Appl. Math. 16 (1963), 331-351.

17. G. Ratcliff, A symbolic calculus for 3-step nilpotent Lie groups, Ph.D. thesis, Yale University, 1983.

18. L. Richardson, Decomposition of the $L^{2}$-space of a general compact nilmanifold, Amer. J. Math. 93 (1971), 173-190.

19. __ Global solvability on compact Heisenberg manifolds, Trans. Amer. Math. Soc. 273 (1982), 309-317.

20. _,$N$-step nilpotent Lie groups with flat Kirillov orbits, Colloq. Math. 52 (1986), 119-121.

21. S. Sternberg, Celestial mechanics. Part II, Benjamin, New York, 1969.

22. G. Warner, Harmonic analysis on semi-simple Lie groups. I, Springer-Verlag, Berlin and New York, 1972.

Department of Mathematics, Louisiana State University, Baton Rouge, LOUISIANA 70803 